RH-08-96

\title{
Scaling and Correlation Functions in a Model of a Two-dimensional Earthquake Fault
}

\author{
Thordur Jonsson \\ Sigurdur Freyr Marinosson \\ Raunvisindastofnun Haskolans, University of Iceland \\ Dunhaga 3, 107 Reykjavik \\ Iceland
}

\begin{abstract}
We study numerically a two-dimensional version of the Burridge-Knopoff model. We calculate spatial and temporal correlation functions and compare their behaviour with the results found for the one-dimensional model. The GutenbergRichter law is only obtained for special choices of parameters in the relaxation function. We find that the distribution of the fractal dimension of the slip zone exhibits two well-defined peaks corresponding to intermediate size and large events.
\end{abstract}

Keywords: Earthquake fault, Burridge Knopoff model, correlation functions

\footnotetext{
${ }^{1} \mathrm{e}-\mathrm{mail}$ : thjons@raunvis.hi.is

${ }^{2} \mathrm{e}-\mathrm{mail}$ : sigurdm@raunvis.hi.is
} 


\section{Introduction}

A mathematical model of an earthquake fault, originally introduced by Burridge and Knopoff [1], has been studied extensively in recent years, see e.g. [2, 3, 4, 5], as an example of a self-organized critical system [6, 7]. The motivation has also been to understand better the mechanism of earthquake generation as well as the search for methods to predict earthquakes [8, 9]. The quantities of main interest have been the scaling law (Gutenberg-Richter law [10]) relating the frequency of earthquakes to their size, other scaling laws as well as spatial and temporal correlations.

In [11, 8] the one-dimensional model is studied in detail by integrating numerically the equations of motion. In [12 a simplified model with qualitatively similar behaviour but simpler time development, corresponding to coupled maps, was introduced. This model, which is analogous to sandpile models [13], was studied in more detail in [4, 14]. Some special two-dimensional models are studied in [5, 15, 16], see 17, 18] for older work in the same vein. The purpose of this article is to generalize the study of [14 to two dimensions and compare the one and the two dimensional cases.

We begin by defining the model and the observables of interest. Then we describe the results of extensive simulations of the size distribution, spatial and temporal correlations as well as the structure of the slip zone. We briefly describe the algorithm, which is essentially the one of [19], and finally we compare our results with previous work and discuss some outstanding questions.

\section{The model}

We describe here the two-dimensional version of the Burridge-Knopoff model and it will be clear from the discussion how to generalize it to higher dimensions. The higher dimensional analogue does of course not have any straightforward physical interpretation. We restrict ourselves to the homogeneous model. The model can be described as a two-dimensional regular array of blocks of equal mass, which slide, subject to a velocity dependent friction, on a plane which can be thought of as one side of an earthquake fault. The blocks are labelled by pairs of integers $(i, j)$, where $i=1, \ldots N_{1}$ and $j=1, \ldots, N_{2}$, such that $x_{i, j}$ is the coordinate of the block labelled by $(i, j)$. Each block is connected to its nearest neighbours in the first coordinate direction by a spring with spring constant $k$ and to its nearest neighbours in the second coordinate direction by leaf springs with a spring constant $k^{\prime}$. We only consider the isotropic case where $k^{\prime}=k$. In addition, each block is connected by a leaf spring, with spring constant $k^{\prime \prime}$, to a sliding plane which lies parallel to the plane of the blocks and moves with velocity $v_{p}$. This plane can be thought of as the other side of the fault. For a discussion of boundary conditions we refer to [16]. For convenience we choose units such that $k^{\prime \prime}=1$ and $v_{p}=1$.

If we start the system at rest in a configuration where the elastic force on each block is smaller than the static friction force, then the blocks remain at rest until the elastic force on one of the blocks exceeds a threshold value $f_{t h}$ which equals the 
static friction. Then this block starts to slide, with increasing velocity to begin with, since the friction is a decreasing function of velocity for small velocities in physically realistic models, see [20]. The motion of the first block may cause the force on some of the neighbouring blocks to exceed threshold so they begin sliding and so on. In some cases the motion of the first block unleashes an avalanche of sliding blocks and this is regarded as a large earthquake. Eventually all the blocks come to rest again, at least if $v_{p}$ is small enough compared to the natural velocities in the spring-block system.

We normalize the coordinates such that $x_{i, j}$ is the displacement of the $(i, j)$ block from its equilibrium position at time $t=0$. The total nonfrictional force $f_{i, j}$ on this block at time $t$ due to the springs is then given by

$$
f_{i, j}=-\left(x_{i, j}-t\right)+k \sum_{(n, m)}\left(x_{n, m}-x_{i, j}\right),
$$

where the sum runs over the four nearest neighbours of the site $(i, j)$. We do not study the Newtonian equations of motion for this system (see [3]) but rather use the simpler discrete dynamics ([12, 5]) which we now describe. The elastic force increases linearly with time on each block, so there is generically a unique first block which reaches the threshold for moving. We let this block move along the first coordinate axis (the direction of the driving force) until the elastic force on it has reached the value

$$
f_{i, j}^{\prime}=\phi\left(f_{i, j}\right)
$$

where $\phi$ is a decreasing function which describes the force relaxation in the system. This motion is assumed to take place in zero time and meanwhile all the other blocks remain at rest. For earthquake dynamics this is an excellent approximation. The slip of the $(i, j)$ block gives rise to an increase in the force on its four nearest neighbours by an amount $\frac{1}{4} \Delta\left(f_{i, j}-\phi\left(f_{i, j}\right)\right)$, where

$$
\Delta=\frac{4 k}{1+4 k}
$$

For a $d$-dimensional system 4 is replaced by $2 d$. In general this force relaxation is modified at the boundary. The parameter $\Delta$ is a measure of the stiffness of the system or, if one prefers, the amount of conservation of elastic stress in the system, $\Delta=1$ corresponding to a conservative system.

In practice we take $\phi$ so that

$$
\phi\left(f_{t h}\right)=f_{t h}-\delta f
$$

where $\delta f>0$ is the smallest possible force relaxation. By making one more choice of units we may assume that $f_{t h}=1$. Using the linear relationship between the coordinates of the blocks and the elastic forces we can easily compute the slips of blocks during earthquakes.

If the force relaxation function $\phi(f)$ decreases fast for $f$ close to $f_{t h}$, then a small slip is easily amplified as we discuss in more detail below. In our simulations we 
follow [3, 12] and use $\phi=\psi_{\alpha}$, where

$$
\psi_{\alpha}(x)=\frac{\left(2 f_{t h}-\delta f\right)^{2}}{\alpha\left(x-f_{t h}\right)+\left(2 f_{t h}-\delta f\right)}-f_{t h}
$$

and $\alpha>0$. Note that $\psi_{\alpha}^{\prime}\left(f_{t h}\right)=-\alpha$ so the size of $\alpha$ is a measure of how easily small slips can trigger large ones. We also discuss briefly the case $\phi=0$ considered in [5, 16] and in more detail in [19].

\section{Observables}

We now describe the quantities that have been of main interest in the study of the model. In addition we introduce a natural notion of a Hausdorff dimension for the slip zone.

If $x_{i, j}$ and $x_{i, j}^{\prime}$ are the coordinates of the blocks before and after an earthquake, its moment is defined as

$$
M=\sum_{i=1}^{N_{1}} \sum_{j=1}^{N_{2}}\left(x_{i, j}^{\prime}-x_{i, j}\right),
$$

There are two other quantities of interest in describing the size of an earthquake, the number of participating blocks which we shall denote by $N$ and the energy $E$ realeased which is obtained by comparing the potential energy in the springs before and after an event.

The magnitude of an earthquake is defined as the logarithm of its "size" so different notions of "size" give rise to different notions of magnitude. In particular, we shall see that the moment magnitude $\mu=\log M$, does not obey a GutenbergRichter law in two dimensions except for very special choices of the parameters.

Before proceeding let us discuss qualitatively the main features of the events observed in the case $\phi=\psi_{\alpha}$. For a soft system the blocks move independently and only make the minimal jumps corresponding to the force relaxation $\delta f$. In fact, the system is periodic when it is sufficiently soft; if a given block moves at a certain time, then all the other blocks move before the given block moves again. At a sharply defined value of $\Delta=\Delta_{0}$ events with more than one block begin to occur in the system and there is no upper bound on the size of events that can occur, see Fig. 1, where this scenario is illustrated for the one-dimensional system. The precise value of $\Delta_{0}$ depends on $\alpha$ and the transition is sharper the larger the value of $\alpha$. In fact our data are consistent with a discontinous change in the size of the largest events for $\alpha>3$. We interpret this transition as being due to the onset of instability in the periodic state. Once this state becomes unstable and the motion of a block can trigger a motion of its neighbours, there is little that prevents earthquakes from spreading over the entire system. As we increase $\Delta$ further the dynamics becomes more chaotic [21], the state of the blocks more irregular and earthquake fronts are more likely to get trapped in "valleys" in the spring-block system. As we increase $\Delta$ still further the size of the maximal events passes through a minimum and eventually the system becomes so stiff that almost any motion of a 
block can trigger a systemwide slip. In Fig. 2 we illustrate the same phenomenon for the two-dimensional system.

Let us discuss this in more detail. The displacement of the first block to move in an earthquake is always

$$
\theta_{1}=\delta f(1-\Delta)
$$

Assuming that all blocks in an infinite system are at threshold the displacement of the $i$-th block in an earthquake is inductively given by

$$
\theta_{i}=1-\Delta+\frac{\Delta}{2} \theta_{i-1}-(1-\Delta) \phi\left(1+\frac{\Delta \theta_{i-1}}{2(1-\Delta)}\right)
$$

This result was first derived for the one-dimensional system in [14] but is in fact independent of the dimensionality of the system. If $\phi$ is a decreasing function, then the sequence $\theta_{i}$ is easily seen to be increasing and bounded so it converges to a value $\theta^{*}$ which is the maximal slip of a single block in an earthquake.

Let us study the stability of the periodic state in a soft system more closely. For simplicity we discuss the one-dimensional case but the mechanism is the same in two and higher dimensions. We assume that the system is in a state where all events are minimal. Then we have for all $i$,

$$
1-\delta f \leq f_{i} \leq 1
$$

where $f_{i}$ is the total force from the springs on block $i$. Let us assume that block 1 is going to move next and $f_{1}=1$. We now also put $f_{2}$ to threshold and we want to derive a sufficient condition on $\alpha$ in $\psi_{\alpha}$ and $\Delta$ so that block 3 will also move. We have

$$
f_{1} \mapsto 1-\delta f
$$

and

$$
f_{2} \mapsto f_{2}^{\prime}=1+\frac{1}{2} \Delta \delta f
$$

Since

$$
1-\delta f \leq f_{3} \leq 1
$$

a sufficient condition for the movement of the 3rd block is

$$
\frac{1}{2} \Delta\left(1-\phi\left(1+\frac{1}{2} \Delta \delta f\right)\right) \geq \delta f .
$$

With $\phi=\psi_{\alpha}$ this condition is equivalent to

$$
\frac{(2-\delta f)^{2}}{\delta f\left(1-\frac{\delta f}{\Delta}\right)}-\frac{4}{\delta f}+2 \leq \alpha \Delta .
$$

Since $\delta f \ll 1$ it follows that the above inequality is roughly equivalent to

$$
4\left(\frac{1}{\Delta}-1\right)+2 \leq \alpha \Delta
$$


which agrees quite well with the location of the onset of the instability in the $\alpha \Delta$ plane, cf. Fig. 1.

We now introduce the correlation functions. Let $F$ be a function of time and the coordinates $x_{i, j}$ of the blocks. We refer to such functions as observables. The average $\langle F\rangle$ of an observable $F$ is defined as

$$
<F>=\lim _{T \rightarrow \infty} \frac{1}{T} \int_{0}^{T} F d t
$$

provided the limit exists. In the simulations we choose of course a finite value of $T$ which must be taken sufficiently large, depending on the observable under study as well as the system size.

We define

$$
\xi_{i, j}(t)=x_{i, j}(t)-v_{p} t
$$

The average $\left\langle\xi_{i, j}>\right.$ is well defined since the long time average of $x_{i, j}$ is proportional to $t$ with coefficient $v_{p}$.

The spatial correlation function $G(i)$ is defined as

$$
G(i)=<\xi_{i+N_{1} / 2, N_{2} / 2} \xi_{N_{1} / 2, N_{2} / 2}>-<\xi_{i+N_{1} / 2, N_{2} / 2}><\xi_{N_{1} / 2, N_{2} / 2}>.
$$

Here we have assumed that $N_{1}$ and $N_{2}$ are even numbers and chosen the center of the system as a reference point in order to minimize finite size effects in the case of free or open boundary conditions. In the case of periodic boundary conditions it does of course not matter which subtraction point we choose. A more general spatial correlation function is obtained by separating the two $\xi$ variables by an arbitrary lattice vector. This more general correlation respects the symmetry of the lattice but does not reveal any features different from those of $G(i)$.

We define the time correlation function (or autocorrelation function) $C(t)$ as

$$
C(t)=\lim _{T \rightarrow \infty} \frac{1}{T} \int_{0}^{T} \xi\left(t^{\prime}+t\right) \xi\left(t^{\prime}\right) d t^{\prime}-<\xi>^{2},
$$

where $\xi=\xi_{N_{1} / 2, N_{2} / 2}$. Clearly $C(0)=G(0)$.

It is of interest to consider the shape of the slip zone in earthquakes. For this purpose we introduce the notion of the Hausdorff dimension $\delta$ of the slip zone. It is defined as

$$
\delta=\frac{\ln N}{\ln D}
$$

where $N$ is the number of blocks that participate in the event and $D$ is the length of the diagonal of the smallest rectangular box, with sides along the coordinate axes, which contains all the blocks that participated in the earthquake. This definition is by no means unique but we find it convenient to work with. All reasonable definitions should be equivalent in the limit of large $N$ and $D$.

\section{Numerical results}

In this section we describe the results of simulations for the relaxation function $\psi_{3}$. Most of the results are for a $200 \times 200$ block system with periodic boundary 
conditions. We begin by considering the size distribution of earthquakes. We let $R(\mu)$ denote the density of events per unit time with moment magnitude $\mu$. There are three regimes in this distribution for all values of $\Delta$ and $\alpha$. We discuss only the case of $\alpha=3$. Other values of $\alpha$ in the range from 2 to 5 do not lead to a qualitatively diferent picture.

For small events the discretization shows clearly up. In the intermediate range from $\mu \approx-2$ to $\mu \approx 0$ the distribution falls off but not linearly except for $\Delta \approx 0.82$. For smaller $\Delta$ the distribution is concave in this region while it is convex for larger values of $\Delta$, see Figs. 3-5. This is quite different from the one-dimensional model where the size distribution is linear to a good approximation in the intermediate range [14]. The distribution of earthquakes as a function of the energy is qualitatively similar. However, the distribution as a function of the number of participating blocks is linear to a very good approximation for $\Delta \geq 0.8$ with a slope which varies continuously with $\Delta$, see Figs. 6-7. For smaller $\Delta$ the distribution becomes concave.

For $\Delta<0.65$ almost all events are minimal but around $\Delta=0.65$ the instability discussed in the previous section sets in, cf. Fig. 2 , and for $\Delta=0.67$ some events extend over the entire system with almost no events of an intermediate size. As $\Delta$ increases further events of intermediate size begin to appear and finally for the very stiff system the peak at large events begins to rise again.

The behaviour of the size distribution is also reflected in the correlation functions. Let us first discuss the spatial correlation $G(i)$, see Fig. 8. For small $\Delta$ we find a random function of small amplitude since the system is periodic. As events involving more than one block begin to occur we find a sharply peaked function at 0 which explodes at the "phase transition" point $\Delta=0.67$ to a broad function whose decay is not very well determined due to finite size effects and the very large fluctuations in the simulation at this value of $\Delta$. For larger values of $\Delta$ the functions narrows until the prevalence of large earthquakes reaches a minimum at $\Delta \approx 0.82$. It broadens again as $\Delta$ is increased further. In an infinite system the correlation should decay monotonically to zero. We see marked finite size effects for $\Delta=0.67$ as well as for $\Delta=0.95$. This is in a qualitative agreement with the behaviour of the spatial correlation in the one-dimensional model and the value of $G(0)$ is roughly proportional to the maximum displacement of blocks in a single earthquake, cf. [14.

The autocorrelation function $C(t)$ is considerably more difficult to calculate in two dimensions than in the one-dimensional case. As far as we can see it has qualitatively the same behaviour in one and two dimensions as long as the system is not too stiff. The correlation decays rapidly with well defined oscillations whose frequency is well explained by the maximum slip of a single block and the driving velocity, see Fig. 9. The data does not allow us to say anything about the decay of the envelope. For a stiff system $(\Delta \approx 0.95)$ the oscillations disappear, a phenomenon not seen in the study of the one-dimensional system.

In the one-dimensional model the structure of the slip zone in an earthquake is always trivial since it is connected. In two dimensions the situation is quite different. The structure of the slip zone is intimately related to the structure of the displacement field $\xi_{i, j}$. In Figs. 10 - 12 we indicate by cross those blocks which are subject to an eleastic force greater than 0.9 at a particular time. The value 0.9 is 
chosen solely for illustrative purposes. We see that the softest system is quite regular and the boundary between the set of blocks which experience a force in excess of 0.9 and the rest of the blocks consistes mostly of straight lines. As we increase $\Delta$ the system becomes more irregular with valleys of all sizes. The system becomes more homogeneous as $\Delta$ is increased still further. The shape of the slip zone for typical earthquakes occuring in systems with these three values of the stiffness parameter are illustrated in Figs. 13 - 15. In the soft system the slip zone has usually a regular boundary, mirroring the structure of the underlying displacement field. The slip zone on Fig. 13 is atypical in the sense that most large earthquakes for $\Delta=0.67$ extend over a large fraction of the system. For intermediate values of $\Delta$ the slip zone becomes more irregular. For the very stiff system, large events tend to extend over most of the system and the slip zone has an irregular boundary. The Hausdorff dimension corresponding to the slip zones in these three cases is clearly smallest for the most chaotic system corresponding to the interemediate value $\Delta=0.82$ for the stiffness parameter.

A further qualitative insight into the dynamics of the system is obtained by following the development of earthquakes in real time and see it move block by block. In the soft system the earthquake front propagates in the beginning along ribbons which make a $45^{\circ}$ angle with the coordinate axes. This is expected because in this way two blocks can cooperate to topple a block which they have as a common nearest neighbour. As the slip zone grows in size it often becomes roughly hexagonal for the largest events. In the stiffest system we also observe the hexagonal expansion of the slip zone but the ribbons are absent and earthquakes engulf the more stuck regions. The intermediate system is most irregular as expected.

The average Hausdorff dimension is not the relevant measure of the structure of the slip zone. This is illustrated in Figs. 16 - 17 for the system with $\Delta=0.67$. Fig. 16 is a histogram of the Hausdorff dimension of events with more than 100 blocks. There are two peaks in the distribution at the values $\delta=1.86$ and $\delta=1.52$. If we look at Fig. 17, which shows the distribution of events with more than 1000 blocks participating, we see that the peak at the lower value of $\delta$ has disappeared. The earthquakes in the $\Delta=0.67$ system therefore fall into two classes: Those that extend over almost the entire system and have a slip zone with straight edges and those that are bounded in size. These two classes of events correspond to the tail and main body of the $R-\mu$ distribution in Fig. 3. The slip zone illustrated in Fig. 13 corresponds to the events that have a Hausdorff dimension close to 2, but most events in this class are in fact much larger.

We observe a similar distribution of the Hausdorff dimension in the stiff system $\Delta=0.95$ with large Hausdorff dimension for the large events whereas the events in the intermediate system $(\Delta=0.82)$ have a Hausdorff dimension that peaks around $\delta=1.6$ and does not depend sensitively on the size cutoff, see Figs. 18 - 21.

\section{The OFC model}

The model studied by by Olami, Feder and Christensen [5], see also [15, 16, 19], is the one we have discussed above with the force relaxation function $\phi=0$. For 
describing real earthquake faults this is probably a highly unrealistic choice of force relaxation since the typical stress drop in earthquakes is believed to be of the order of $10 \%$ or less [22]. In this model the size distribution (defined as the logarithm of the number of participating blocks) falls nicely on a straight line. Other notions of size do not yield a linear distribution.

In order to compare the OFC model with the one we have simulated we have calculated the spatial as well as the temporal corelation functions, see Figs. 22 and 23. They are qualitatively quite similar to the ones found in the Nakanishi model with the main difference that the autocorrelation function does not oscillate around zero but around a positive value, see Fig. 23. This might very well be a finite size effect which are known to make the analysis of the OFC model quite subtle [19].

\section{The algorithm}

In order to simulate the temporal behaviour of an observable $X$ we have to run the system for a certain time $T$ in order to obtain resonable statistics. In most cases we are not interested in finite size effects and want to have the system as large as possible. It is therefore important how the computational time scales with $N$ when $T$ is constant. For many parameter values the average size of an event is not a function of $N$ and therefore the generation of an earthquake is an $O(1)$ algorithm and the average time between earthquakes scales like $N^{-1}$. To run the dynamics in an $N$ block system for a time $T$ we therefore need a number of earthquakes proportional to $T N$. If we had an $O(1)$ method of finding the block closest to threshold we could carry out the simulation in a time $O(N)$. Using a method introduced by Grassberger [19 this possible under the assumption that the distribution of the forces on the blocks has no delta-function peaks. Our method is quite similar. We use $M$ boxes $B[0], B[1], . ., B[M-1], M \propto N$, which can store blocks in doubly-linked lists. The advantage of having a doubly-linked list is that the removal of a block from such a list is an $O(1)$ operation. The indexing of the $B$ 's is understood modulo $M$. We define $\epsilon=\frac{2}{M-1}$ and $q_{i}=f_{i}-t$ for $i=1,2, . ., N$, where $t$ is the physical time. In our implementation we also have two other boxes $A_{1}$ and $A_{2}$ which can hold blocks. They are used to keep track of the blocks that go over threshold. We need two such boxes because we must keep track of the order in which the blocks move in an earthquake. The idea is to keep the blocks in the $B$ boxes ordered in such a way that the block closest to threshold can be found in a time $O(1)$.

In the simulations reported in this paper we use a global relaxation function $\phi$ and a global $\Delta$, corresponding to a homogeneous system. With trivial modifications it is possible to use the same algorithm where each block $i$ has its own relaxation function $\phi_{i}$ satisfying $\left|\phi_{i}\right|<1$ and the springs between the blocks have different spring constants.

In the beginning we set $t=0$ and distribute the $f_{i}$ 's randomly $-1<f_{i}<1$. We

put block $i$ in $B\left[\left\lfloor\frac{1-q_{i}}{\epsilon}\right\rfloor\right]$, where $\lfloor x\rfloor$ denotes the largest integer smaller than $x$. The boxes $A_{1}$ and $A_{2}$ are empty. Then we perform the following steps consecutively: 
1. All the blocks are experiencing force between -1 and $1, t \geq 0$, and for $i=$ $1,2, . ., N$ block $i$ is in $B\left[j_{i}\right]$ where $j_{i}=\left\lfloor\frac{1-q_{i}}{\epsilon}\right\rfloor$. This implies

$$
-1-t<q_{i} \leq 1-t
$$

and then

$$
\left\lfloor\frac{t}{\epsilon}\right\rfloor \leq j_{i} \leq\left\lfloor\frac{t}{\epsilon}\right\rfloor+M-1
$$

and

$$
1+t-\left(j_{i}+1\right) \epsilon<f_{i} \leq 1+t-j_{i} \epsilon
$$

for all $i$. From this it follows that if the box $B\left[\left\lfloor\frac{t}{\epsilon}\right\rfloor\right]$ is not empty, then it contains the block $i_{\max }$ closest to threshold. If it is empty, we look into the boxes $B\left[\left\lfloor\frac{t}{\epsilon}\right\rfloor+1\right], B\left[\left\lfloor\frac{t}{\epsilon}\right\rfloor+2\right],$. until we find a nonempty box, which then contains $i_{\max }$. We find the block closest to threshold and remove it from the box. We define $f_{\max }=f_{i_{\max }}$ and $\delta t=1-f_{\max }$. We then increase $t$ by $\delta t$. This does not affect the ordering in the boxes for the $q$ 's will not change. After this $f_{\max }$ is equal to 1 and we label the $i_{\max }$ block $i_{\text {active }}$.

2. We update the $i_{\text {active }}$ block according to the dynamics, i.e.

$$
f_{i_{\text {active }}} \mapsto \phi\left(f_{i_{\text {active }}}\right)
$$

and update $q_{i_{\text {active }}}$ accordingly. Then we put it into the box $B\left[\left\lfloor\frac{1-q_{i_{\text {active }}}}{\epsilon}\right\rfloor\right]$ and remove all its neighbours $i_{x}$ from their boxes. Because we can adress them through their positions in the block-array this is an $O(1)$ operation. We modify their $f$ 's according to

$$
f_{i_{x}} \mapsto f_{i_{x}}+\frac{1}{2 D} \Delta\left(f_{i_{\text {active }}}-\phi\left(f_{i_{\text {active }}}\right)\right),
$$

where $D$ is the dimension of the system and update their $q$ 's. If a neighbour becomes critical, i.e. has force above 1 , we put it into the box $A_{2}$. The rest is put back in the $B$ boxes, $i_{x}$ in $B\left[\left\lfloor\frac{1-q_{i_{x}}}{\epsilon}\right\rfloor\right]$.

3. We look into the box $A_{1}$. If it is not empty we remove some block $i$ from $A_{1}$, set $i_{\text {active }}=i$ and go back to step 2 . If the box $A_{1}$ is empty we go to the box $A_{2}$. If $A_{2}$ is not empty we remove some block $i$ from it and set $i_{\text {active }}=i$, move the rest of the blocks in $A_{2}$ to $A_{1}$, and go back to step 2. If both $A_{1}$ and $A_{2}$ are empty, then there are no more critical blocks at this time, i.e. the earthquake is over, and we go back to step 1.

This completes our description of the algorithm.

\section{Discussion}

In this paper we have generalized the simulation of the Nakanishi model to two dimensions. We have not been able to go much beyond systems of size $200 \times 200$ 
so our results have considerable finite size effects which are not straightforward to entangle. In general these systems do not have any translationally invariant thermodynamic limit as we saw clearly in the case of a stiff one-dimensional system in [14]. The simulations reported here also show that when the system makes the transition from the trivial periodic state when the blocks move independently of each other there is a very abrupt change with systemwide events, both in the oneand the two-dimensional models.

The only qualitative difference we have found between the one- and the twodimensional cases is the absence of any linear region in the $R-\mu$ distribution in two dimensions and the positivity of the autocorrelation function for a stiff twodimensional system.

We tried to find a transition from a one-dimensional behaviour, as one expects in a long and narrow system, to properly two dimensional behaviour. However, finite size effects did not allow us to see such a crossover clearly. In real earthquakes, the frequence size distribution is expected to be governed by a two-dimensional theory for small events but by a one-dimensional theory for large events for which the seismogenic zone extends through the litosphere [23]. By a careful analysis of an elongated two-dimensional model we should be able to see such a transition in the model discussed here.

Acknowledgement. This work was supported by the Research Fund of the University of Iceland, the Icelandic Science Foundation and Nyskopunarsjodur Namsmanna.

\section{References}

[1] R. Burridge and L. Knopoff, Model and theoretical seismicity, Bull. Seismol. Soc. Am., 57 (1967) 341 - 371.

[2] J. M. Carlson, J. S. Langer and B. E. Shaw, Dynamics of earthquake faults, Rev. Mod. Phys. 66 (1994) 657 - 670.

[3] J. M. Carlson and J. S Langer, Mechanical model of an earthquake fault, Phys. Rev. A 40 (1989) 6470 - 6484.

[4] H. Nakanishi, Statistical properties of the cellular-automaton model for earthquakes, Phys. Rev. A 43 (1991) 6613 - 6621.

[5] Z. Olami, H. J. S. Feder and K. Christensen, Self-organized criticality in a continuous, nonconservative cellular automaton modeling earthquakes, Phys. Rev. Lett. 68 (1992) 1244 -1247.

[6] P. Bak, C. Tang and K. Wiesenfeld, Self-organized criticality: An explanation of $1 / f$ noise, Phys. Rev. Lett. 59 (1987) 381 - 384.

[7] P. Bak and C. Tang, Earthquakes as a self-organized critical phenomenon, J. Geophys. Res. 94 (1989) 15635 - 15637. 
[8] J. M. Carlson, Time intervals between characteristic earthquakes and correlations with smaller events: An analysis based on a mechanical model of a fault, J. Geophys. Res. 96 (1991) 4255 - 4267.

[9] S. L. Pepke, J. M. Carlson and B. E. Shaw, Prediction of large events on a dynamical model of a fault, J. Geophys. Res. 99 (1994) 6769 - 6788.

[10] B. Gutenberg and C. F. Richter, Seismicity of the earth, Princeton University Press, Princeton (1954).

[11] J. M. Carlson, J. S. Langer, B. E. Shaw and C. Tang, Intrinsic properties of a Burridge-Knopoff model of an earthquake fault, Phys. Rev. A 44 (1991) 884 897.

[12] H. Nakanishi, Cellular-automaton model of earthquakes with deterministic dynamics, Phys. Rev. A 41 (1990) 7086 - 7089.

[13] Y. C. Zhang, Phys. Rev. Lett. 63 (1989) 470.

[14] T. Jonsson and S. F. Marinosson, Correlation functions and power spectra in a model of an earthquake fault, Phys. Lett. A (1995) 165-172.

[15] Z. Olami and K. Christensen, Temporal correlations, universality, and multifractality in a spring-block model of earthquakes, Phys. Rev. A 46 (1992) R1720 - R1723.

[16] K. Christensen and Z. Olami, Scaling, phase transitions, and nonuniversality in a self-organized critical cellular automaton model, Phys. Rev. A 46 (1992) $1829-1838$.

[17] M Otsuka, A simulation of earthquake occurrence, Phys. Earth Planet. Interiors, 6 (1972) $311-315$.

[18] S. R. Brown, C. H. Scholz and J. B. Rundle, A simplified spring-block model of earthquakes, Geophys. Res. Lett. 18 (1991) 215 - 218.

[19] P. Grassberger, Efficient large-scale simulations of a uniformly driven system, Phys. Rev. E (1994) $2436-2443$.

[20] B. N. J. Peterson and E. Tosatti (Ed.), Physics of sliding friction. NATO ASI Series E Vol. 311, Kluwer, Dordrecht (1996).

[21] A. Crisanti, M. H. Jensen, A. Vulpiani and G. Paladin, Strongly intermittent chaos and scaling in an earthquake model, Phys. Rev. A 46 (1992) R7363 R7366.

[22] C. H. Scholz, The mechanics of earthquakes and folding. Cambridge University Press, Cambridge, 1990.

[23] J. F. Pacheco, C. H. Scholz and L. R. Sykes, Changes in frequency-size relationship from small to large earthquakes, Nature 355 (1992) 71 - 73. 


\section{Figure Caption}

Fig. 1. The figure illustrates, as a function of $\Delta$, the average number of blocks participating in the 10 largest out of $2 \times 10^{7}$ events in a one dimensional system consisting of 10,000 blocks for values of $\alpha$ ranging from 2 to 5 .

Fig. 2. The figure illustrates, as a function of $\Delta$, the average number of blocks participating in the 10 largest out of $10^{8}$ events in a $200 \times 200$ block system for $\alpha=3$.

Fig. 3. The figure shows the relative frequency of earthquakes as a function of their magnitude for $\Delta=0.67$.

Fig. 4. The figure shows the relative frequency of earthquakes as a function of their magnitude for $\Delta=0.82$.

Fig. 5. The figure shows the relative frequency of earthquakes as a function of their magnitude for $\Delta=0.95$.

Fig. 6. The figure shows the relative frequency of earthquakes as a function of the number of participating blocks for $\Delta=0.82$.

Fig. 7. The figure shows the relative frequency of earthquakes as a function of the number of participating blocks for $\Delta=0.95$.

Fig. 8. The spatial correlation function for 3 different values of $\Delta$.

Fig. 9. The autocorrelation function for 3 different values of $\Delta$.

Fig. 10. The crosses indicate those blocks that are subject to an elastic force greater than 0.9 in a $200 \times 200$ block system with $\Delta=0.67$.

Fig. 11. The crosses indicate those blocks that are subject to an elastic force greater than 0.9 in a $200 \times 200$ block system with $\Delta=0.82$.

Fig. 12. The crosses indicate those blocks that are subject to an elastic force greater than 0.9 in a $200 \times 200$ block system with $\Delta=0.95$.

Fig. 13. The crosses indicate the position of the blocks that participated in a typical earthquake in a $200 \times 200$ block system with $\Delta=0.67$.

Fig. 14. The crosses indicate the position of the blocks that participated in a typical earthquake in a $200 \times 200$ block system with $\Delta=0.82$.

Fig. 15. The crosses indicate the position of the blocks that participated in a typical earthquake in a $200 \times 200$ block system with $\Delta=0.95$.

Fig. 16. A histogram illustrating the distribution of the Hausdorff dimension of the slip zone for events consisting of more than 100 blocks in a system with $\Delta=0.67$.

Fig. 17. A histogram illustrating the distribution of the Hausdorff dimension of the slip zone for events consisting of more than 1000 blocks in a system with $\Delta=0.67$.

Fig. 18. A histogram illustrating the distribution of the Hausdorff dimension of the slip zone for events consisting of more than 100 blocks in a system with $\Delta=0.82$. 
Fig. 19. A histogram illustrating the distribution of the Hausdorff dimension of the slip zone for events consisting of more than 1000 blocks in a system with $\Delta=0.82$.

Fig. 20. A histogram illustrating the distribution of the Hausdorff dimension of the slip zone for events consisting of more than 100 blocks in a system with $\Delta=0.95$.

Fig. 21. A histogram illustrating the distribution of the Hausdorff dimension of the slip zone for events consisting of more than 1000 blocks in a system with $\Delta=0.95$.

Fig. 22. The spatial correlation function in the OFC model with $\Delta=0.8$.

Fig. 23. The autocorrelation function in the OFC model with $\Delta=0.8$. 


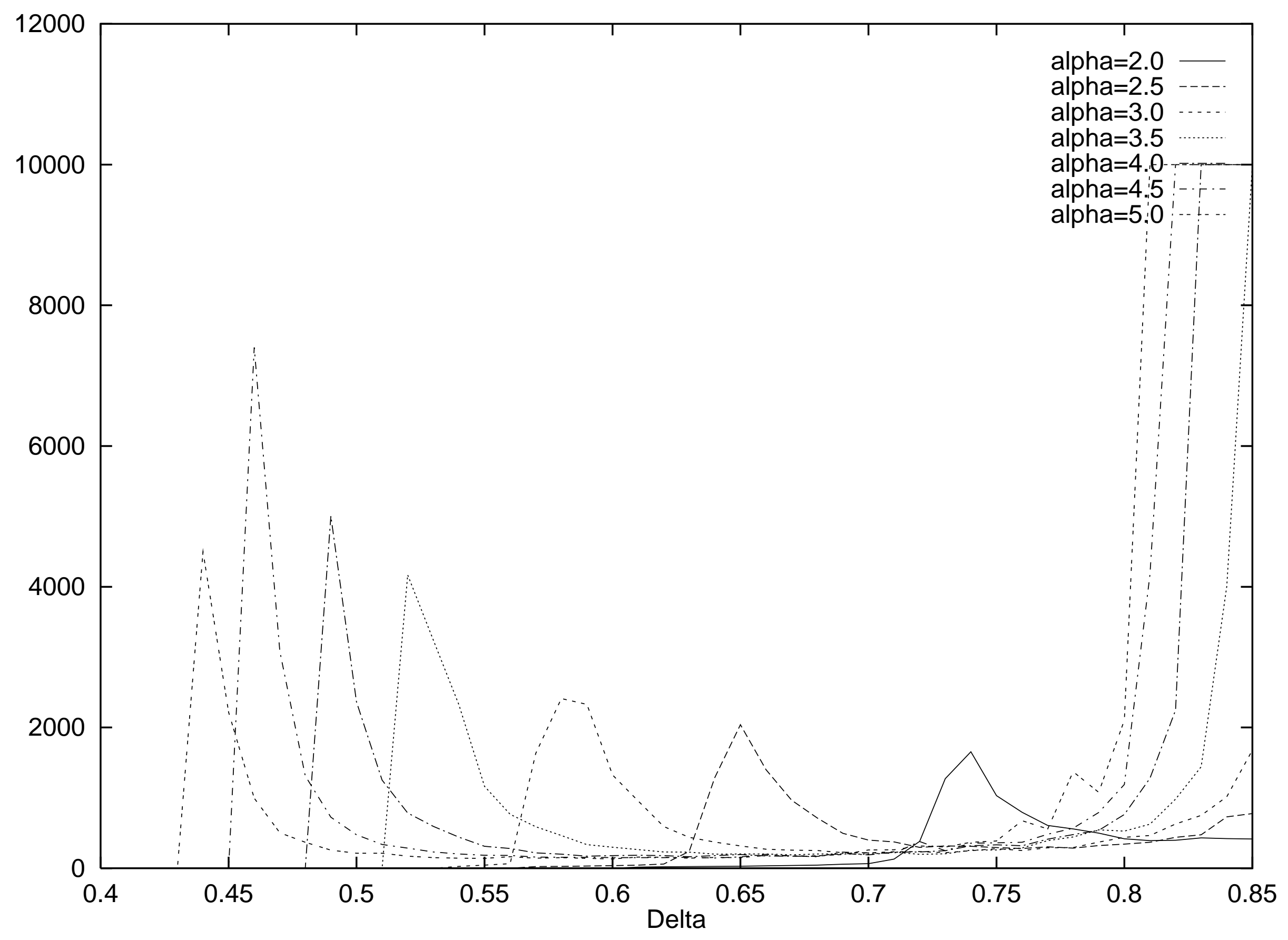




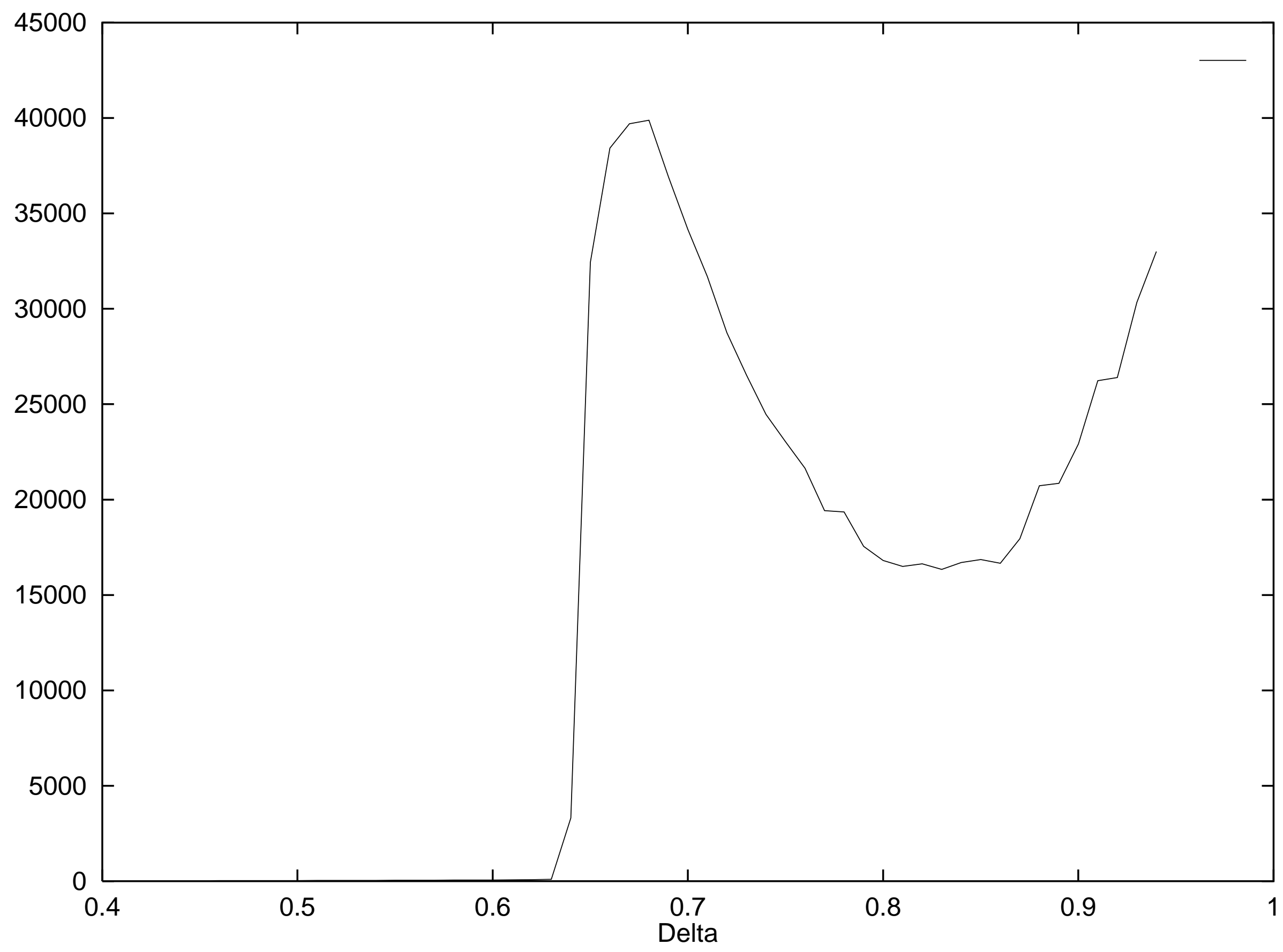




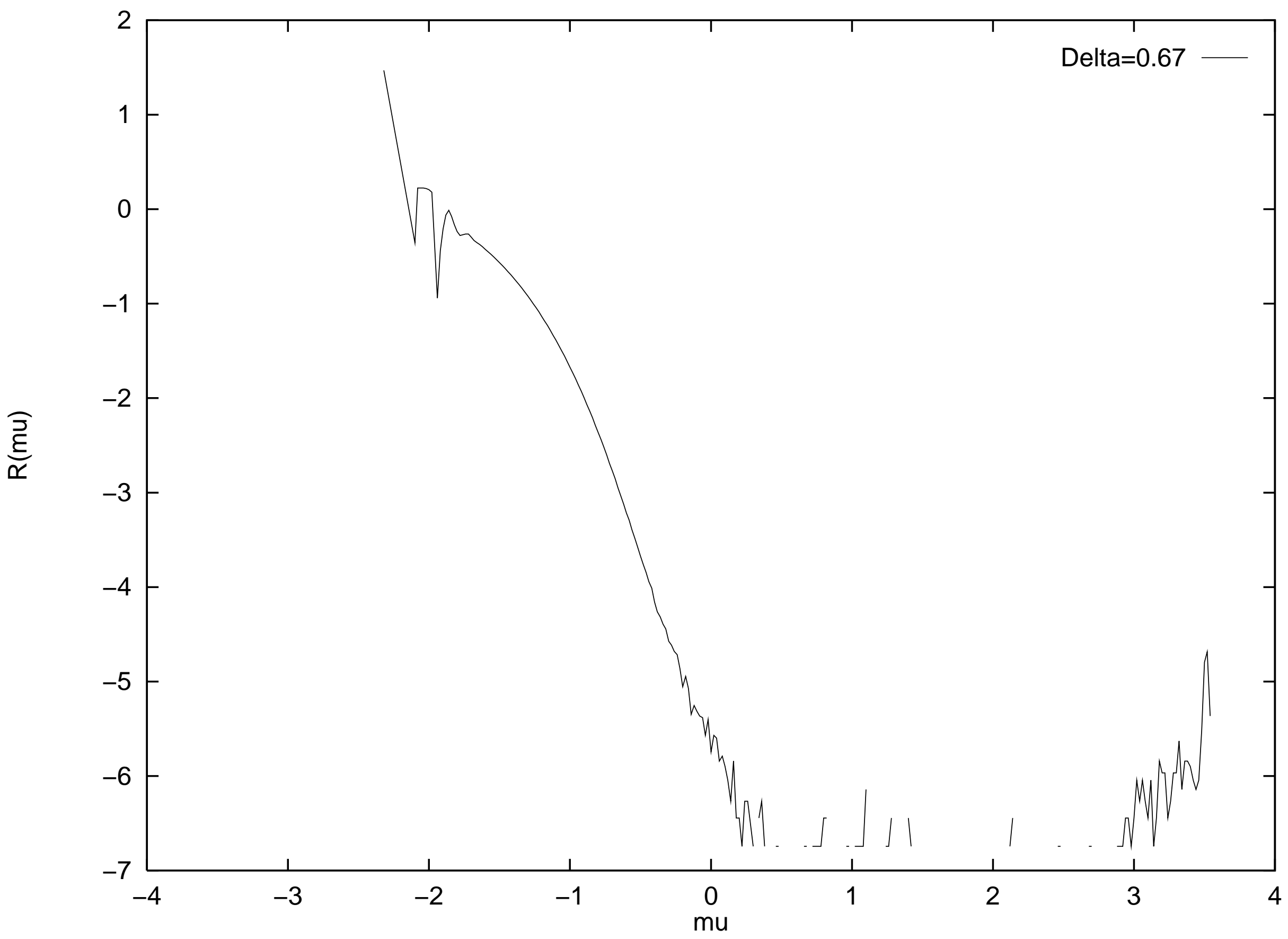




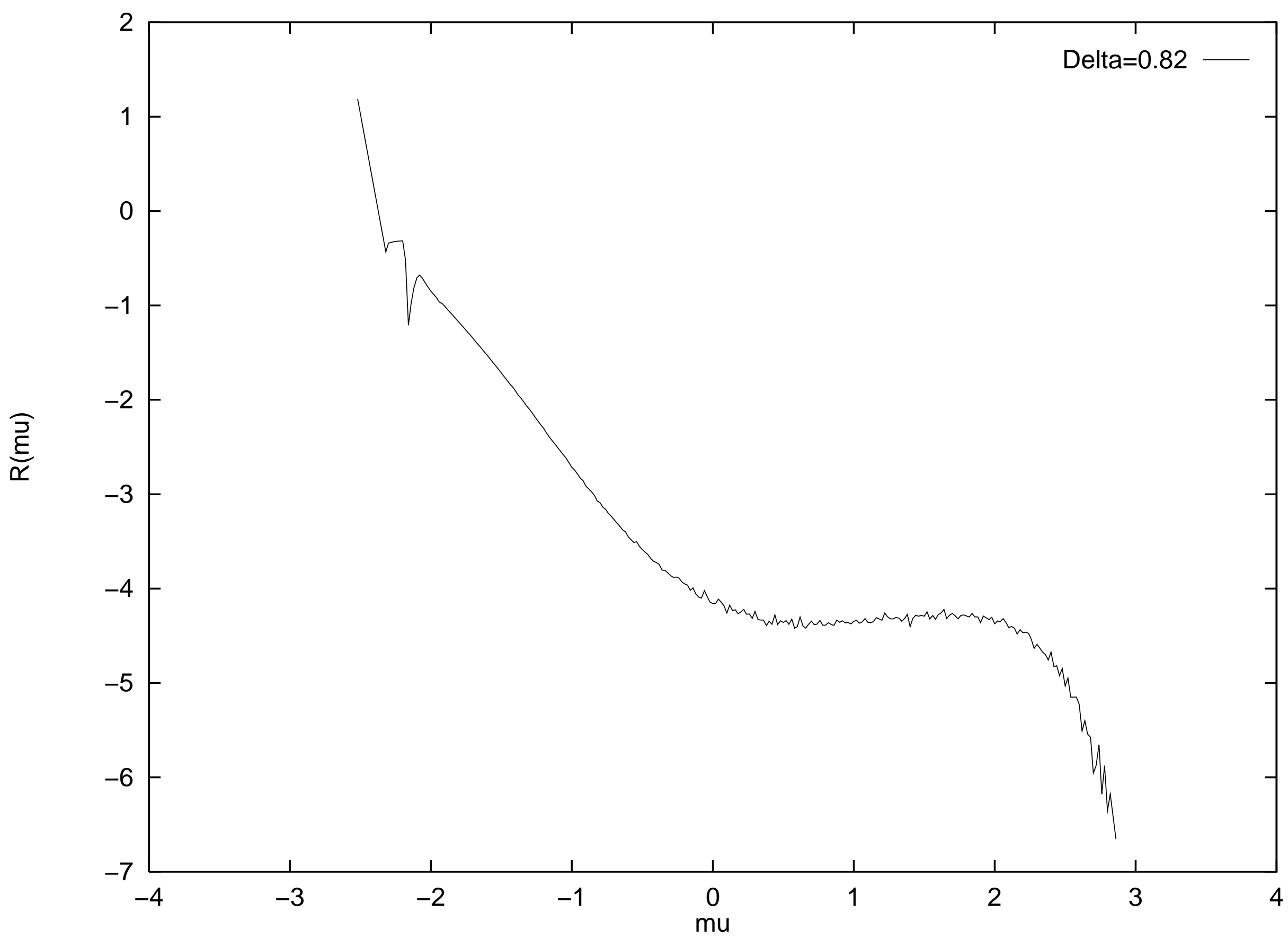




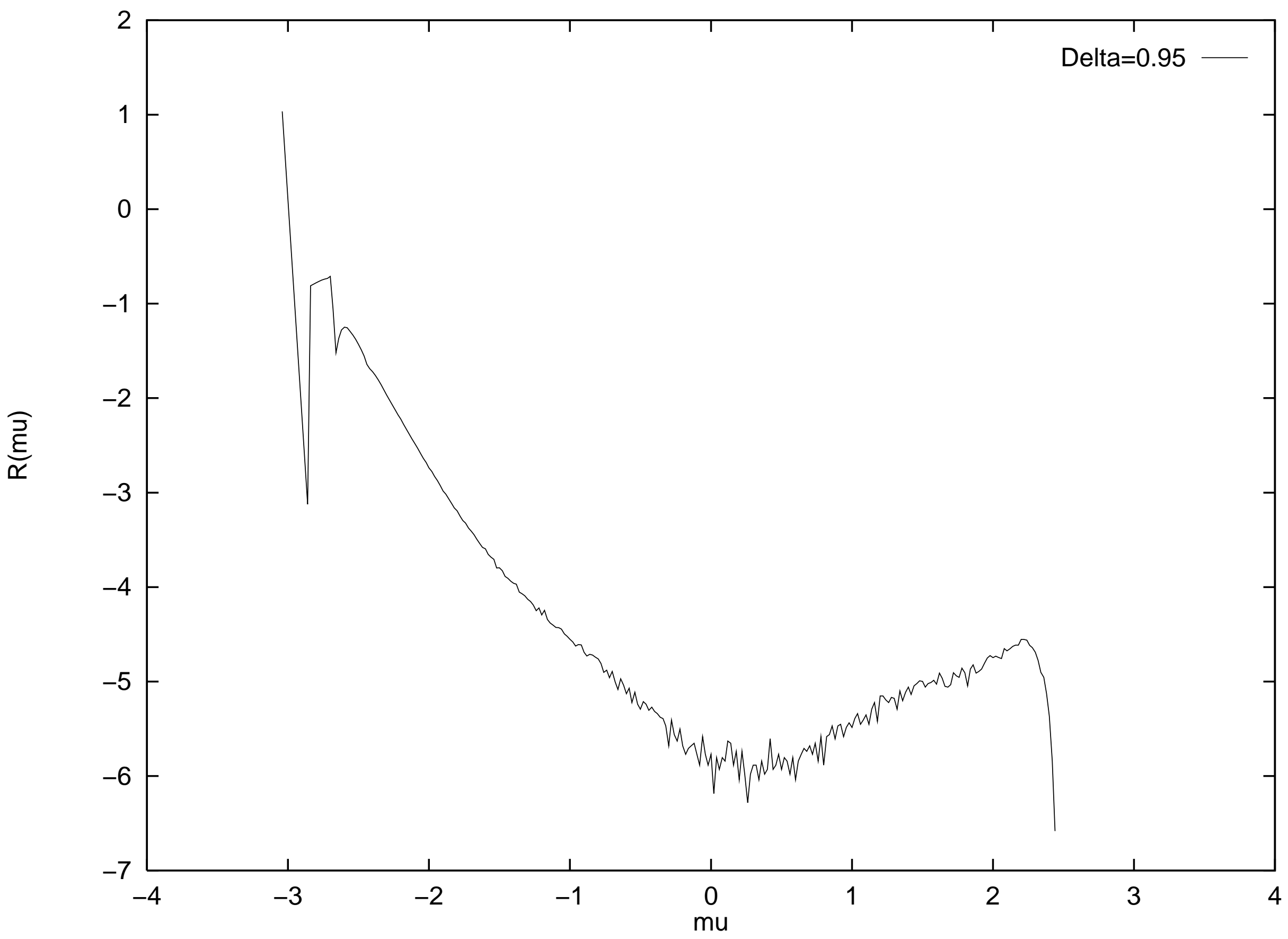




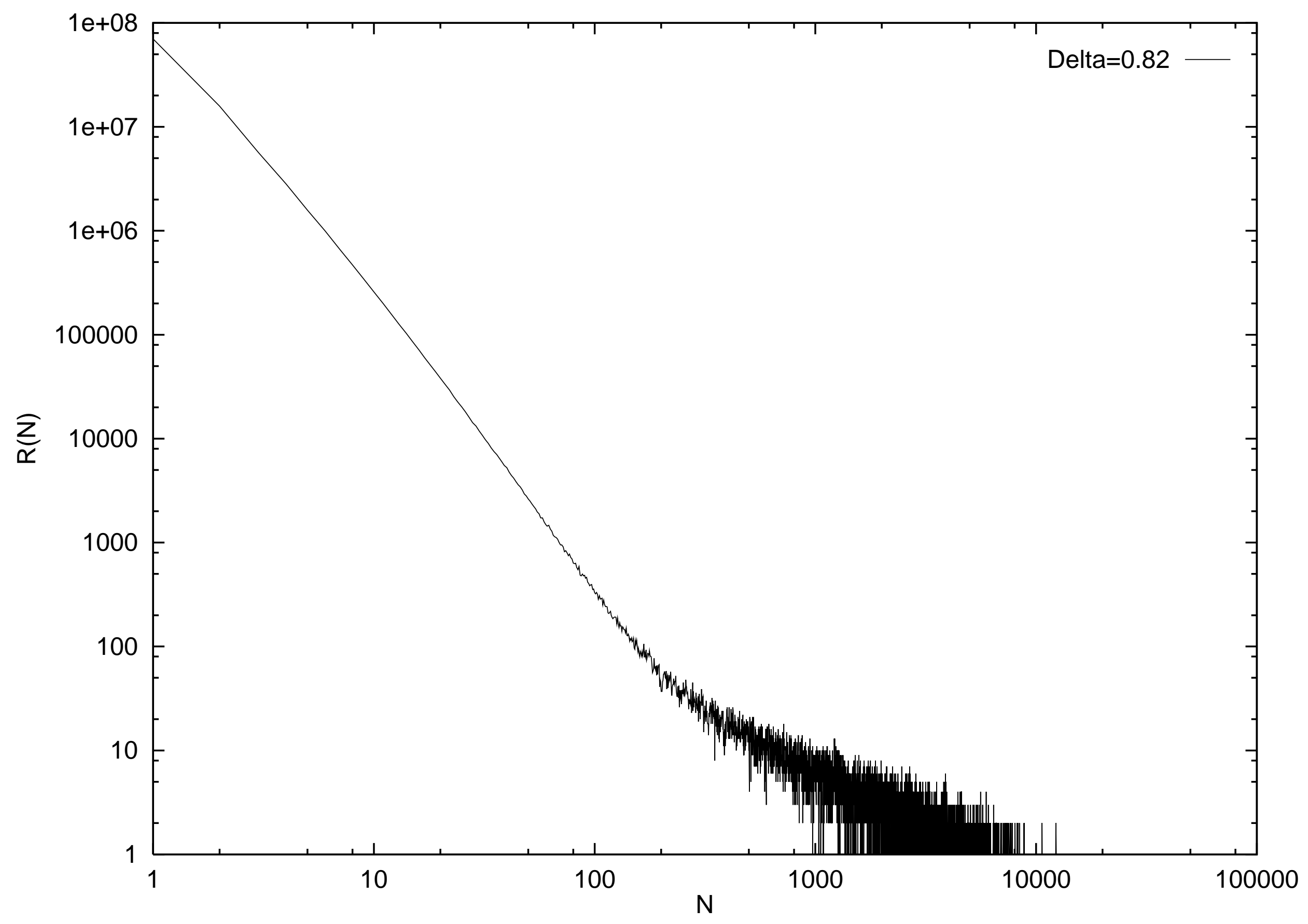




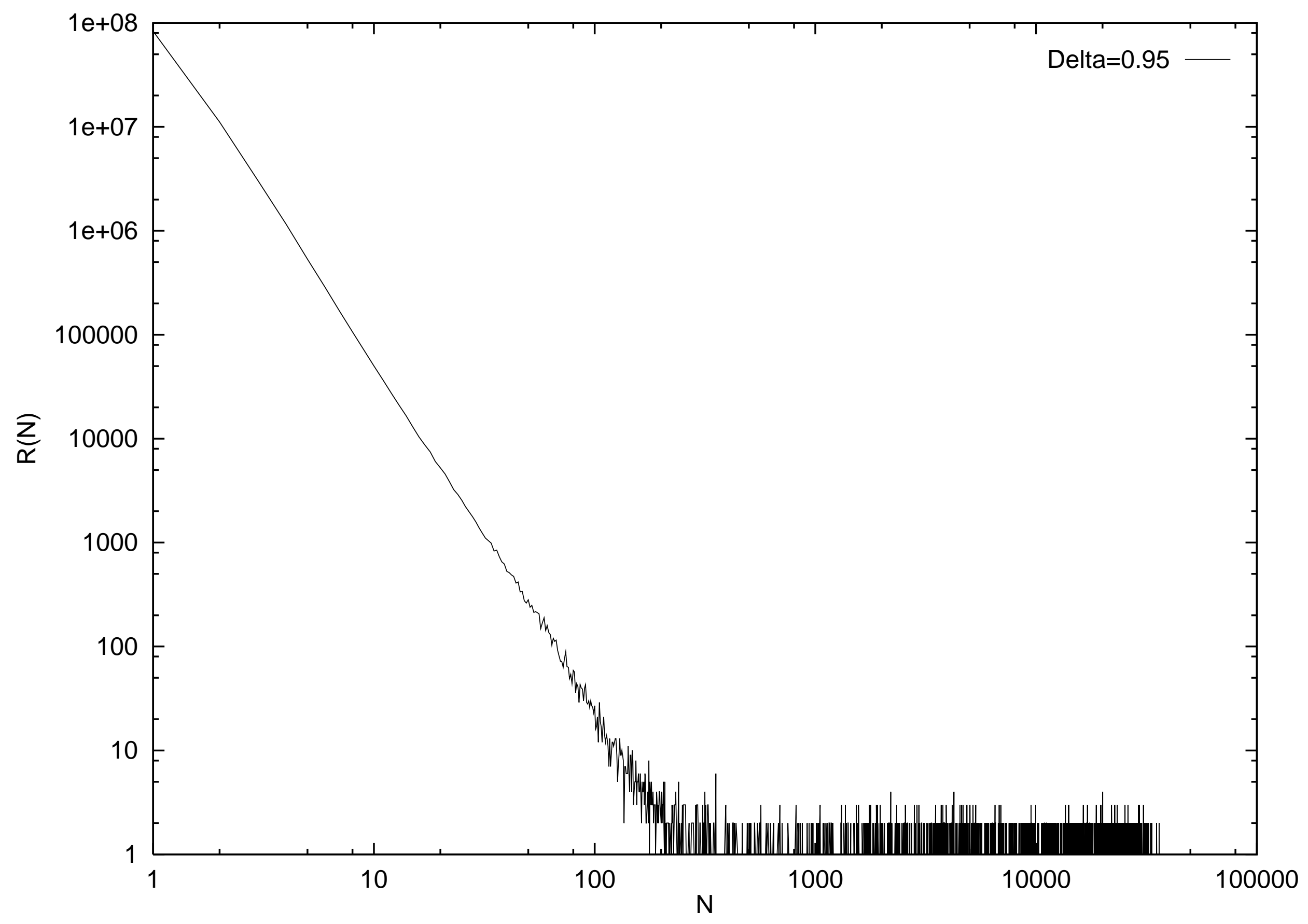




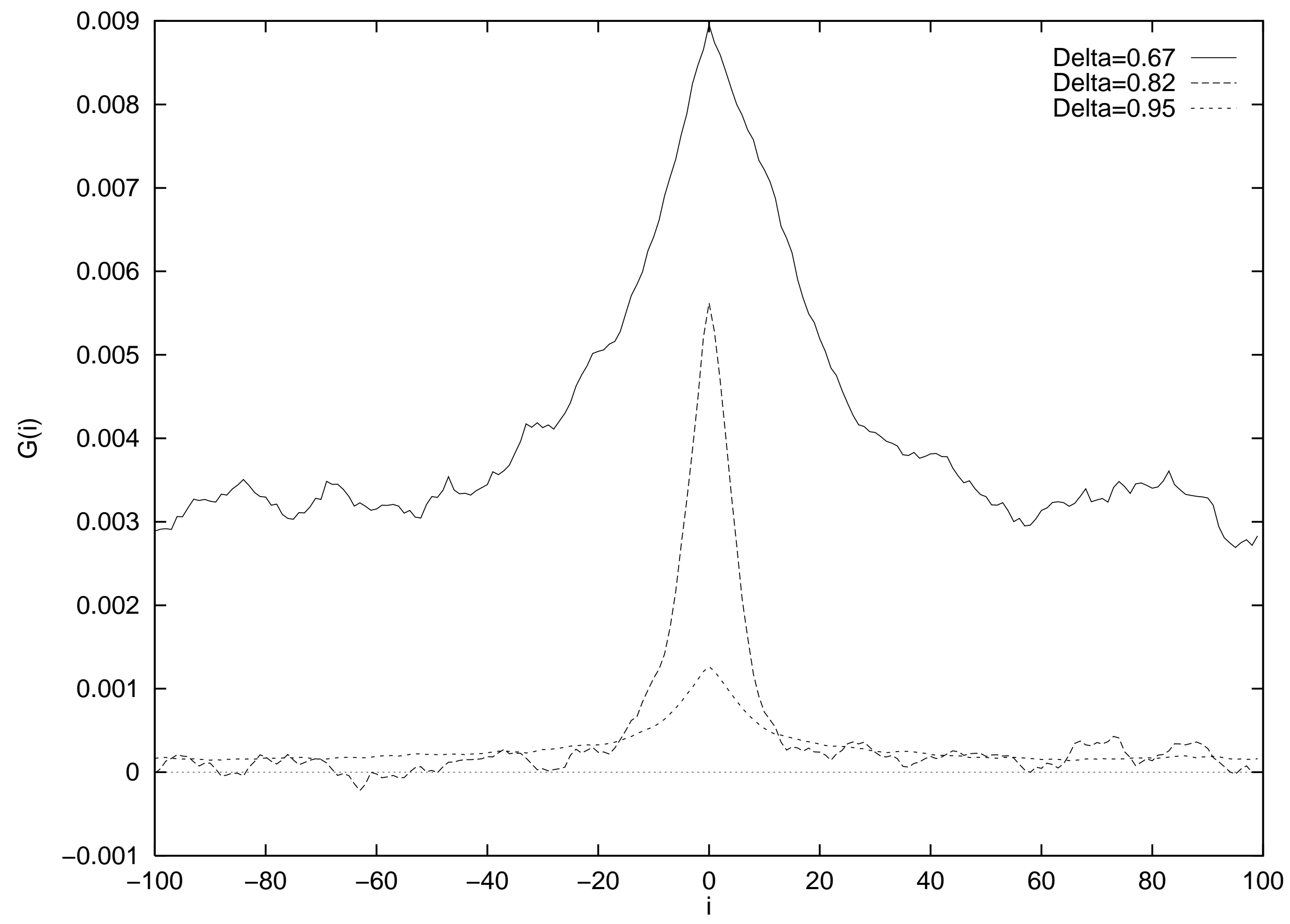




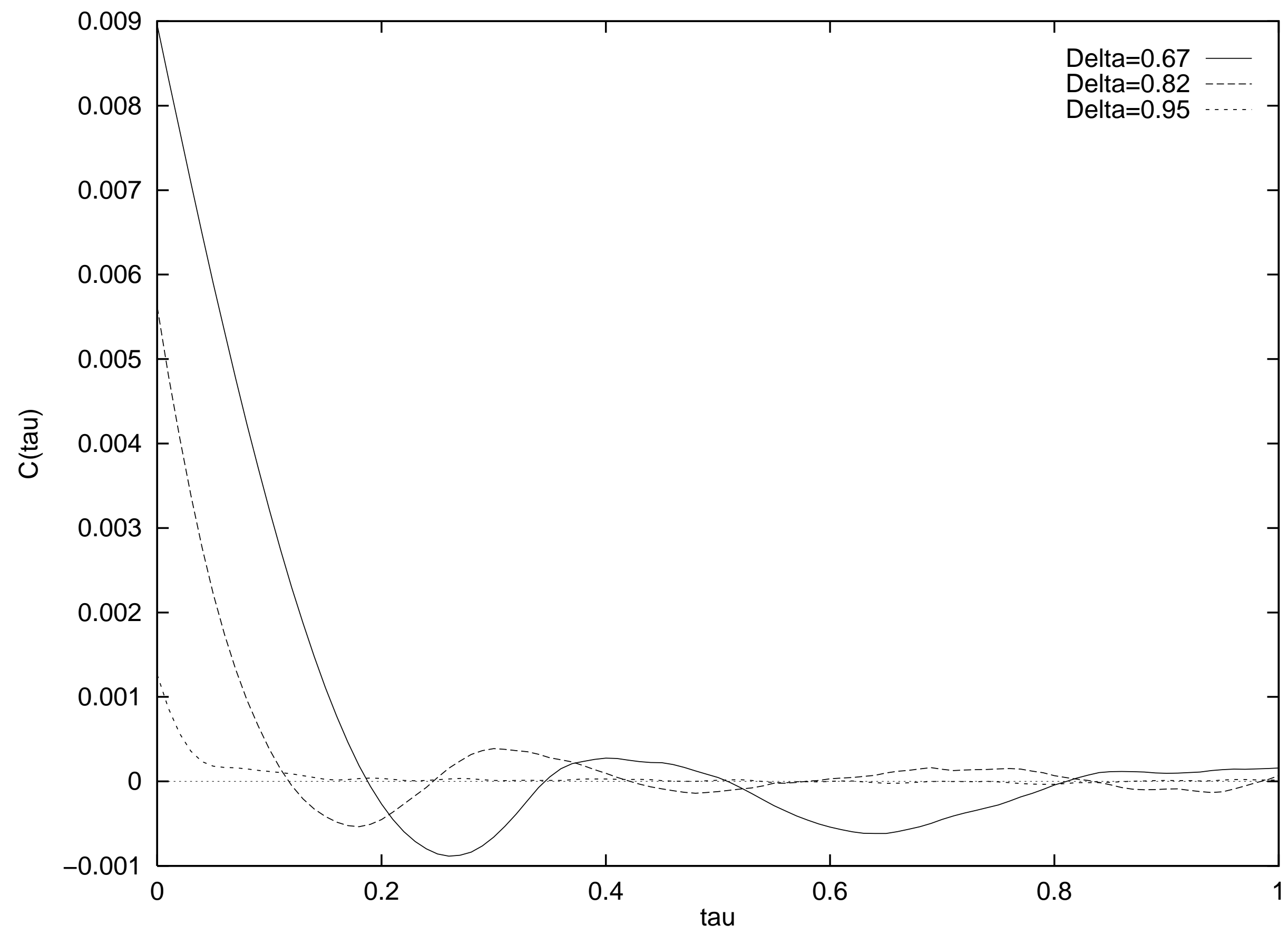


$\mathrm{f}>0.9$, Delta $=0.67$

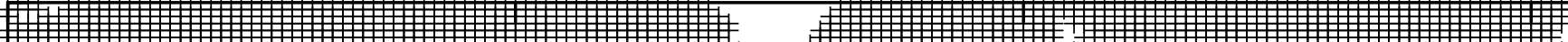

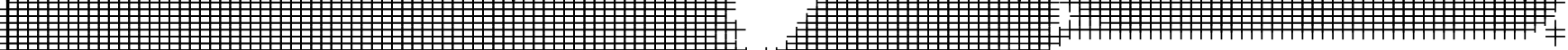

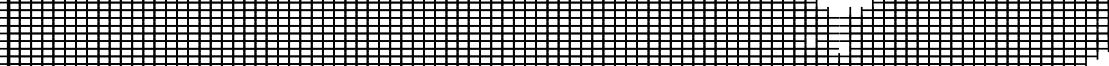

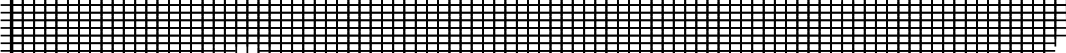
弗 弗 弗

弗

150 蓆

150 业

1 (1)

100 19

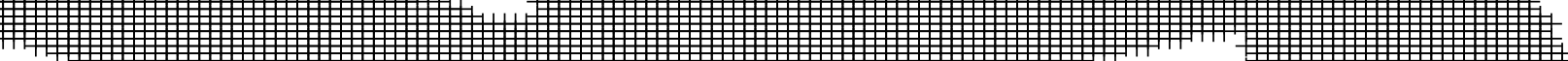

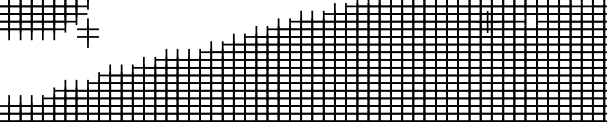

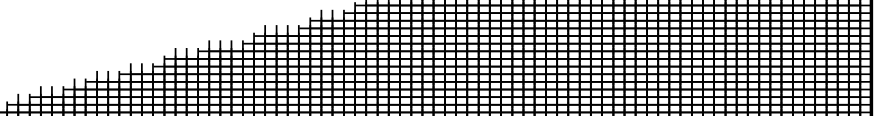

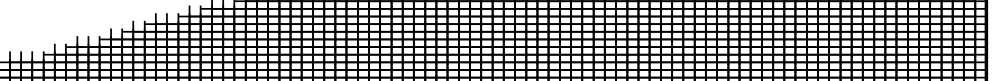

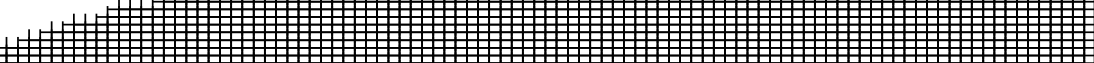

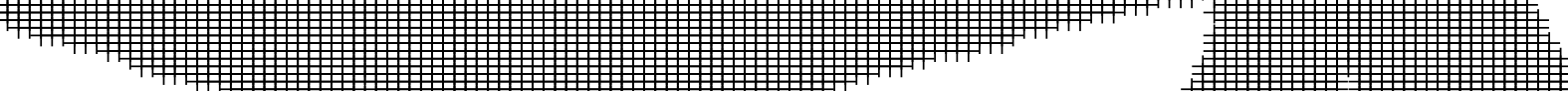

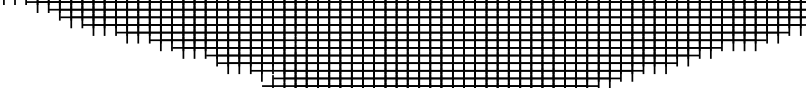
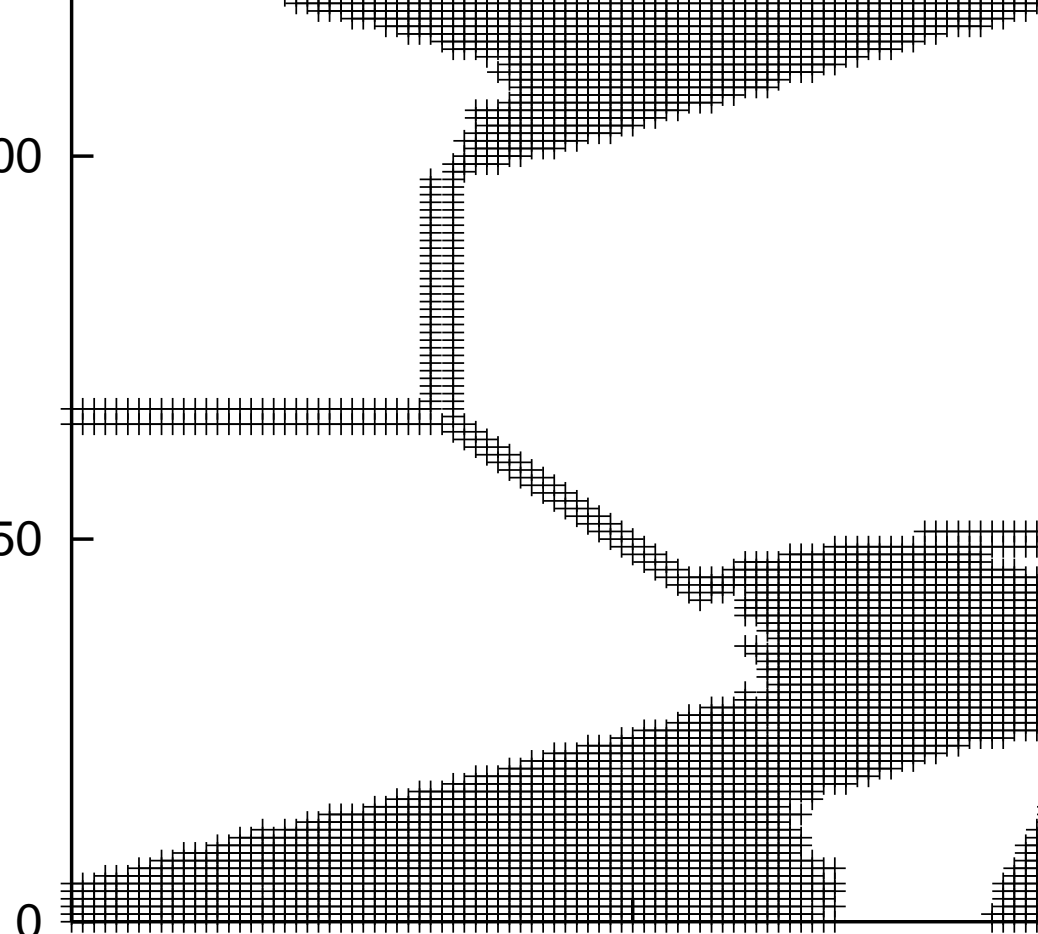

0

50

100

150 


\section{$\mathrm{f}>0.9$, Delta $=0.82$}

200

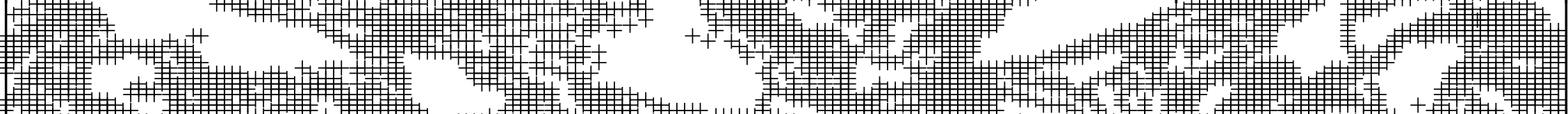

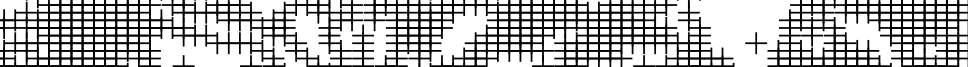

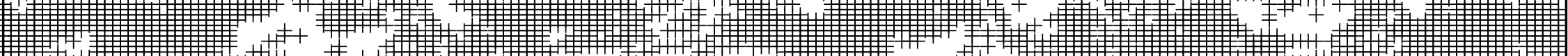
芩地 L

150

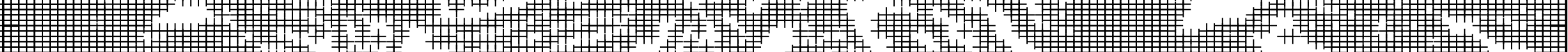
率 W

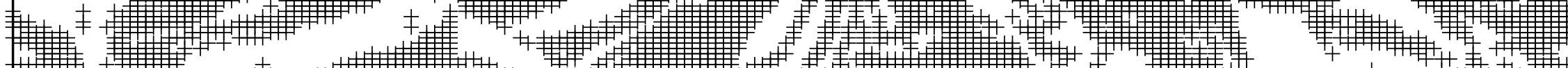
廉

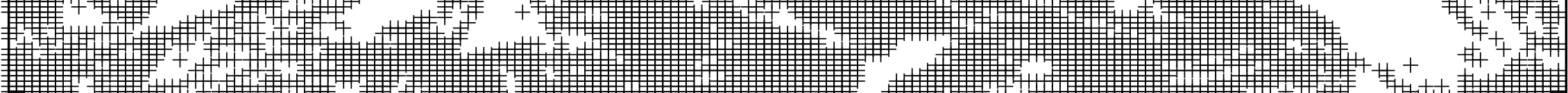

100 $\begin{array}{llllll}1 & 1\end{array}$

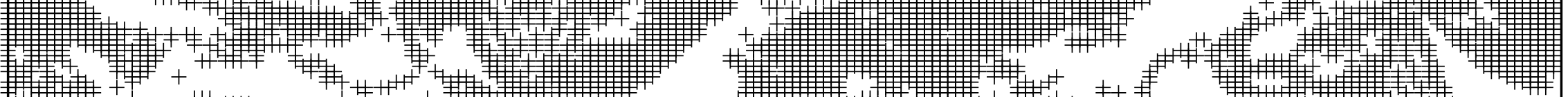

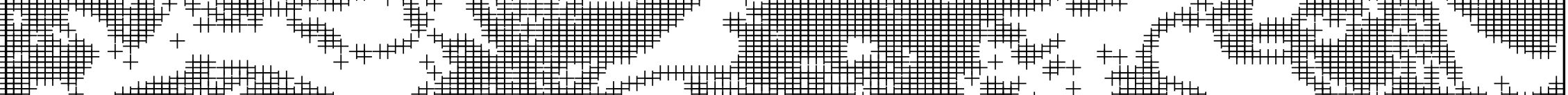

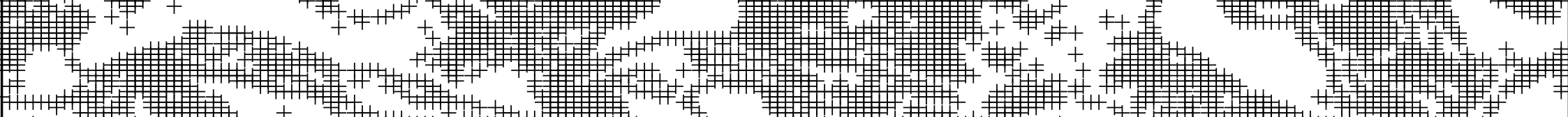

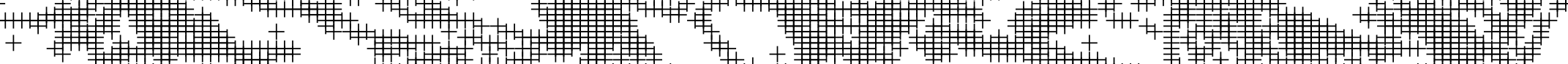

$50 \mathrm{C}$

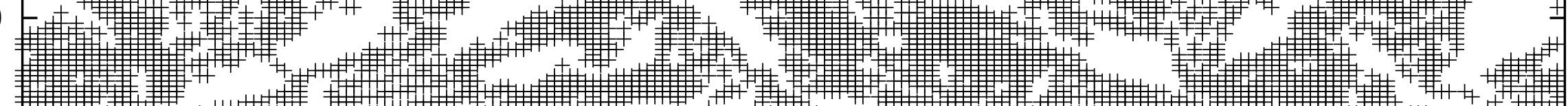

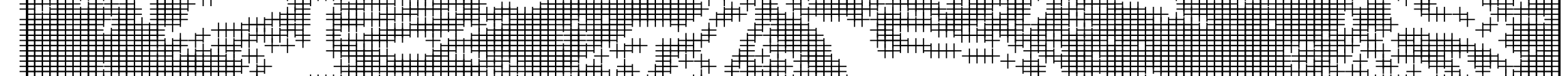

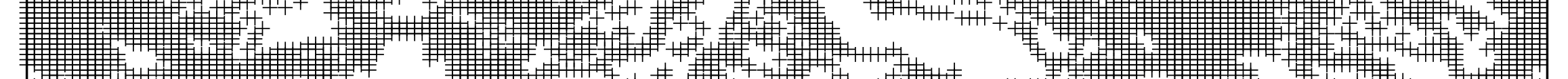

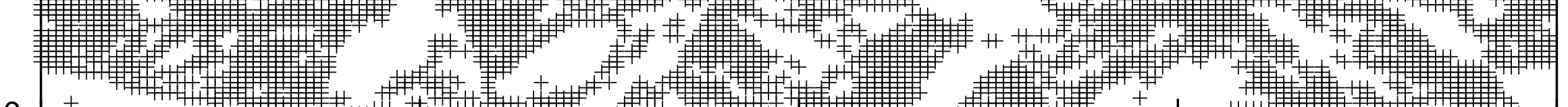

0

0

50

100

150 
$\mathrm{f}>0.9$, Delta $=0.95$

200

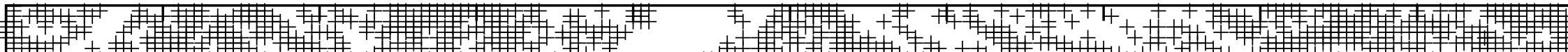

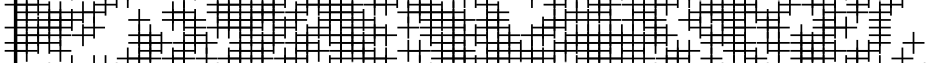

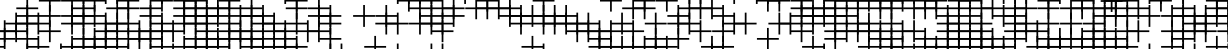

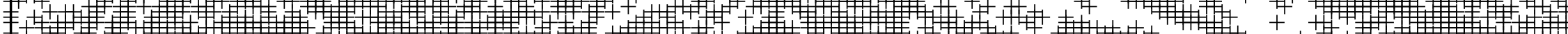

$180 \mathrm{Y}$

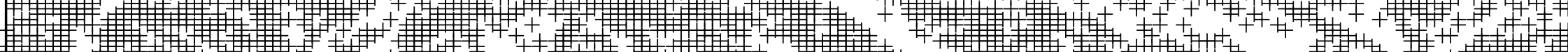

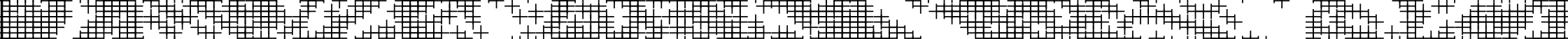

160

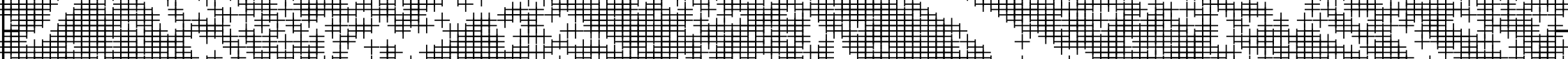

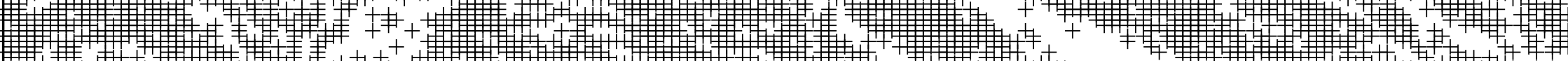
4+

140 皮 皮

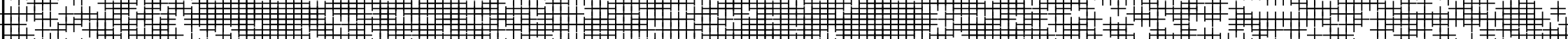
亦

120

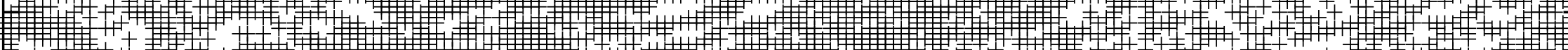

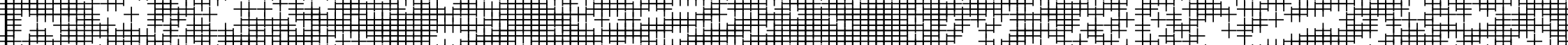

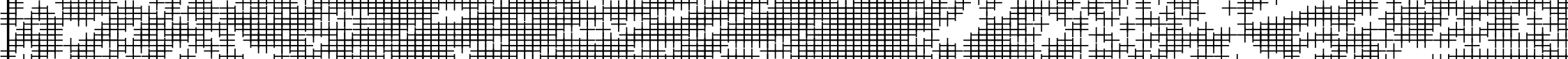

$100 \mathrm{Y}$

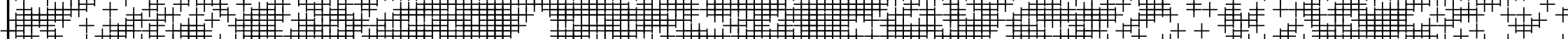
( I

80 I

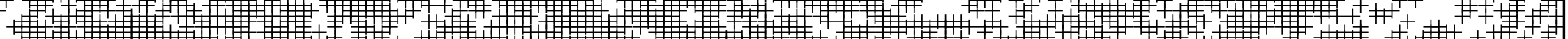

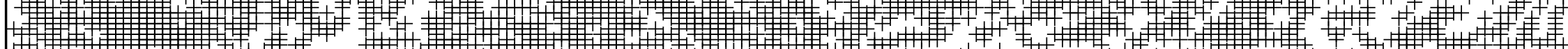

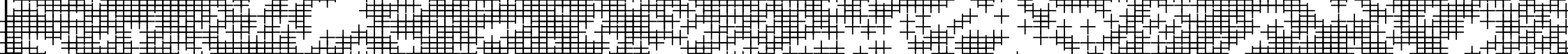

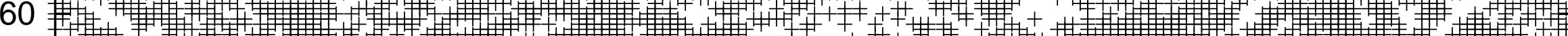

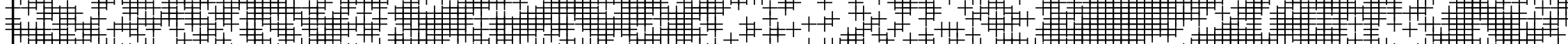

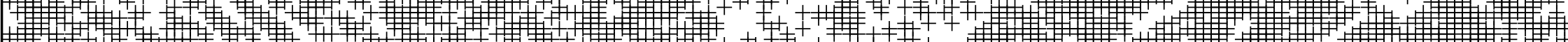

40 圱

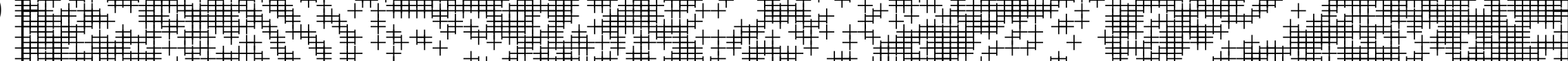

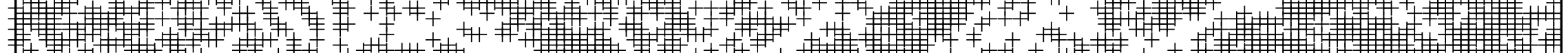

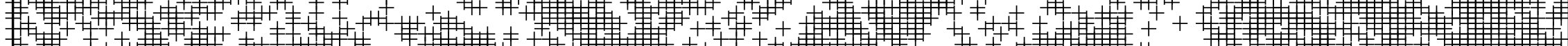

20 基

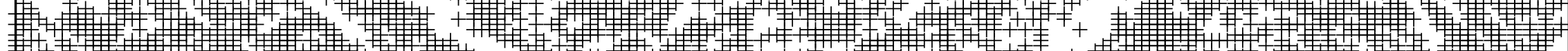

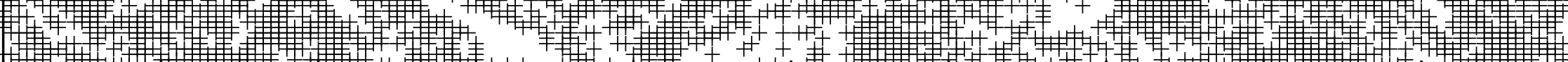

0

20

40

60

80

100

120

140

160

180

200 


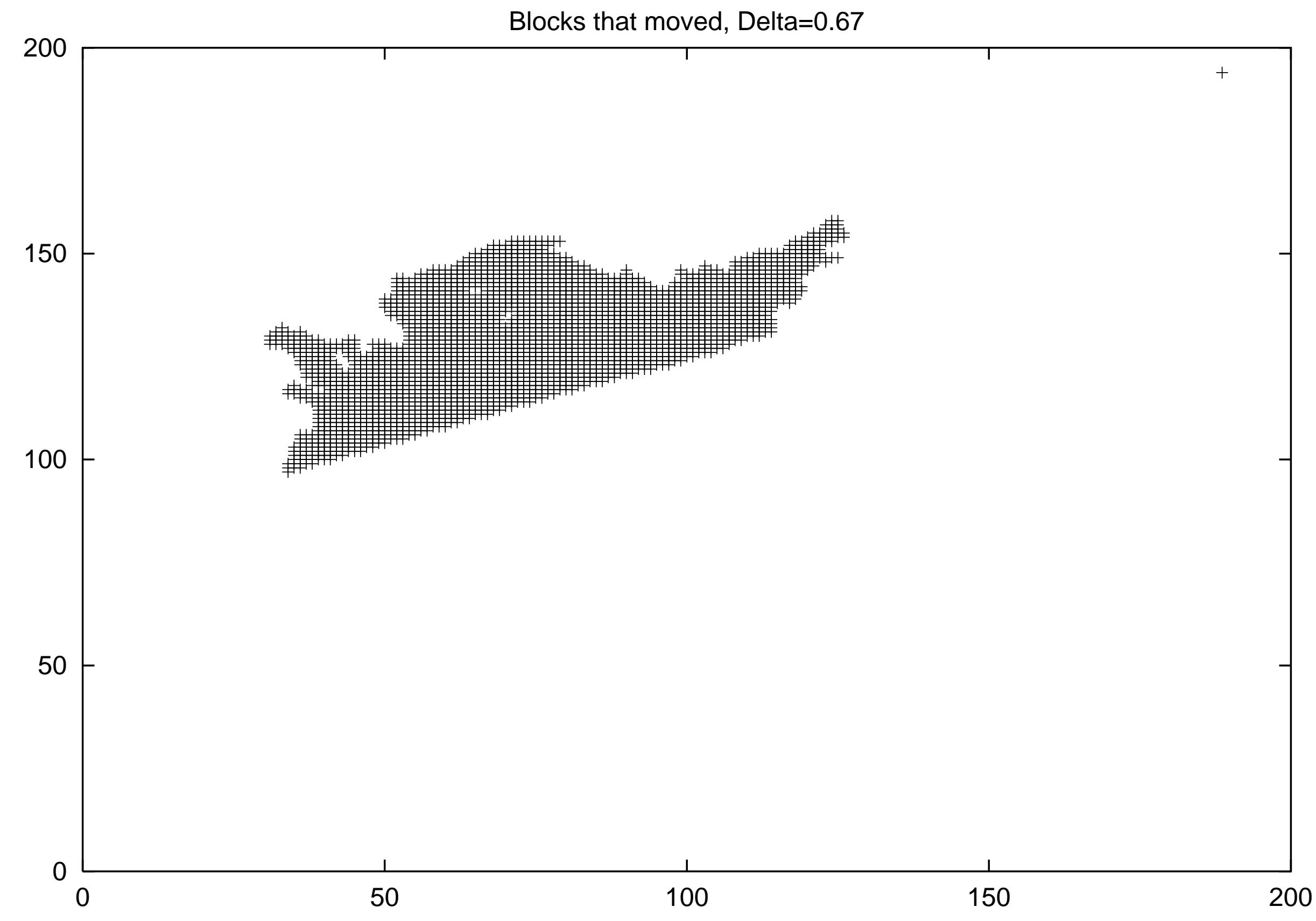


Blocks that moved, Delta $=0.82$

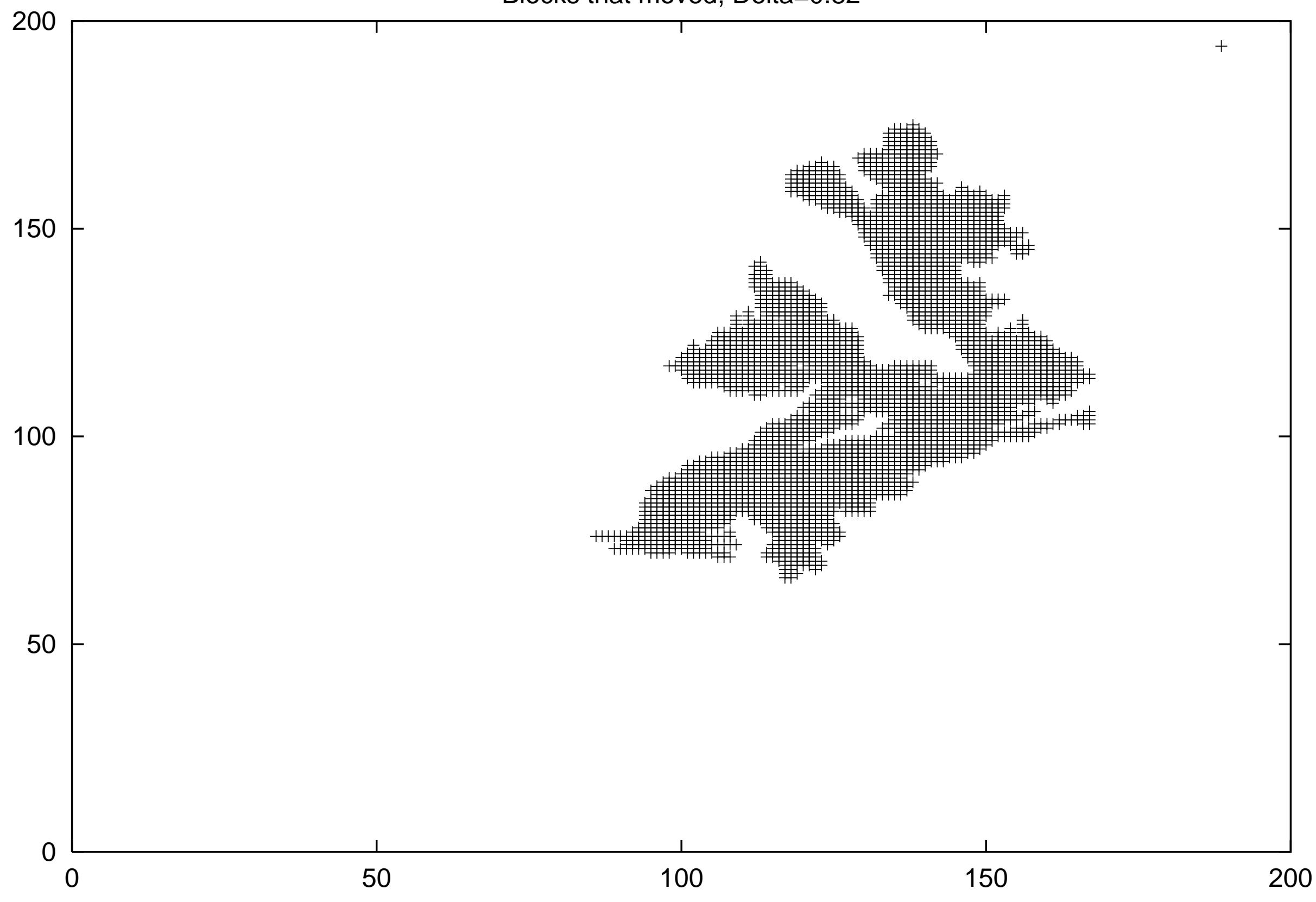


Blocks that moved, Delta $=0.95$

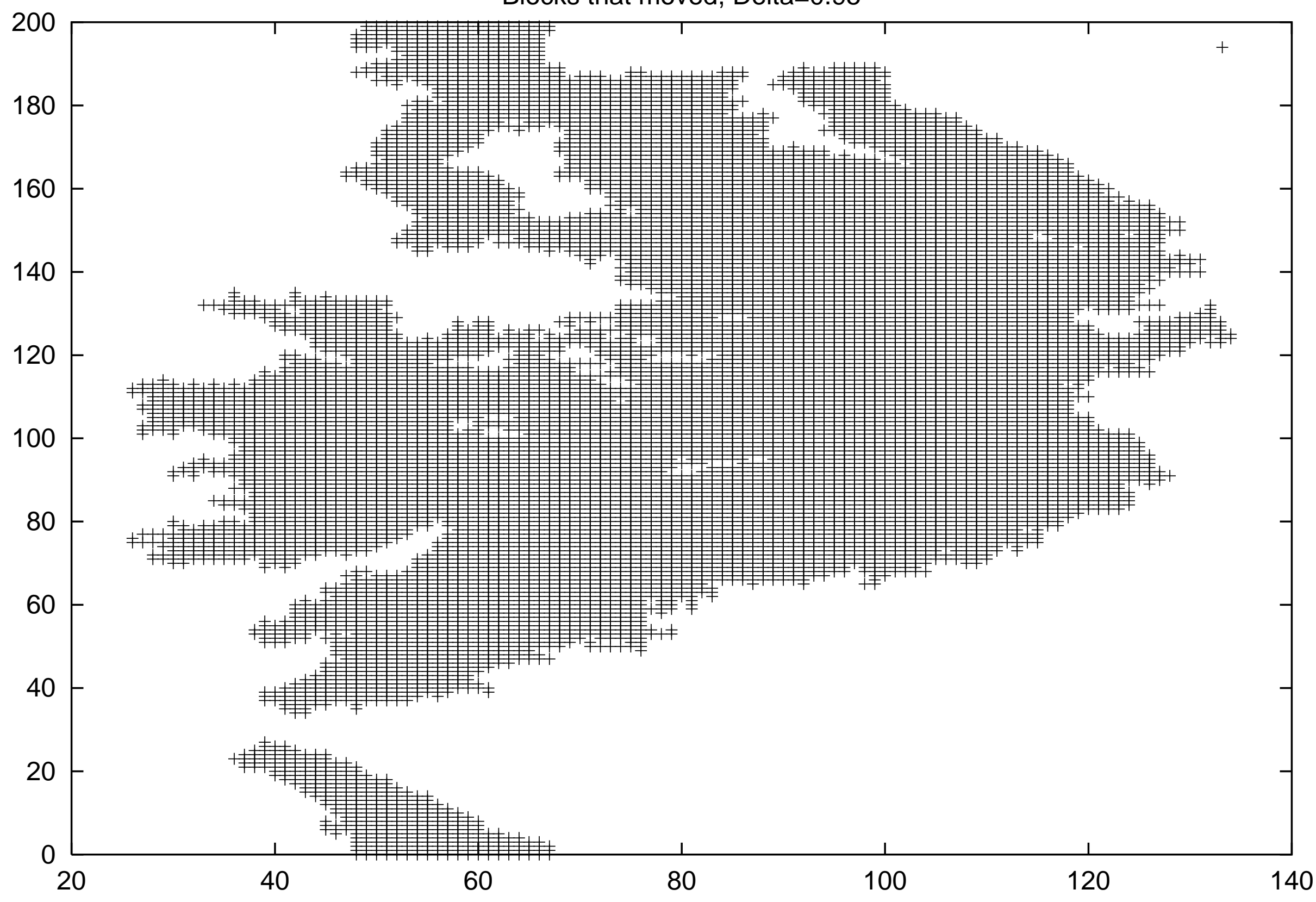




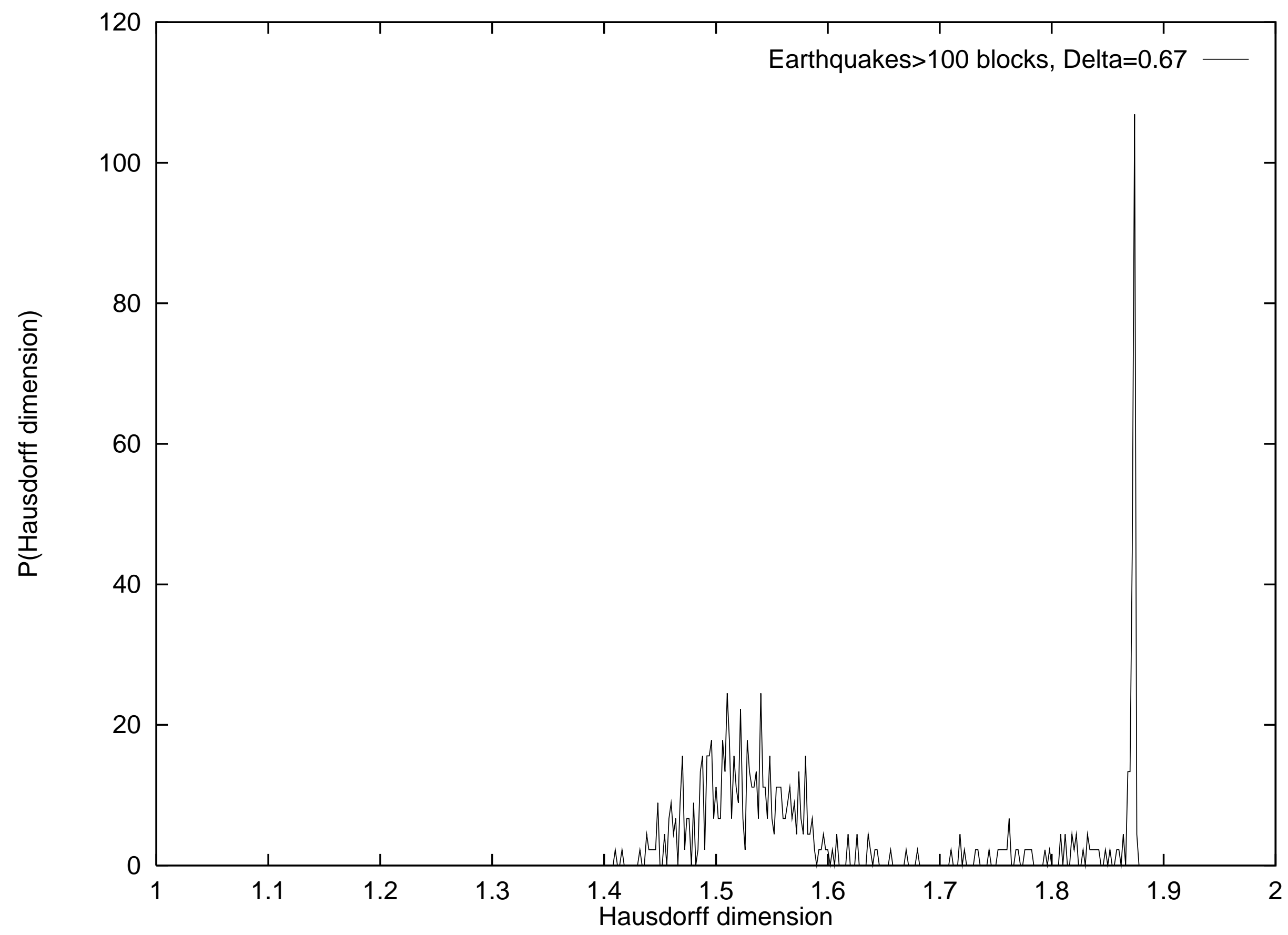




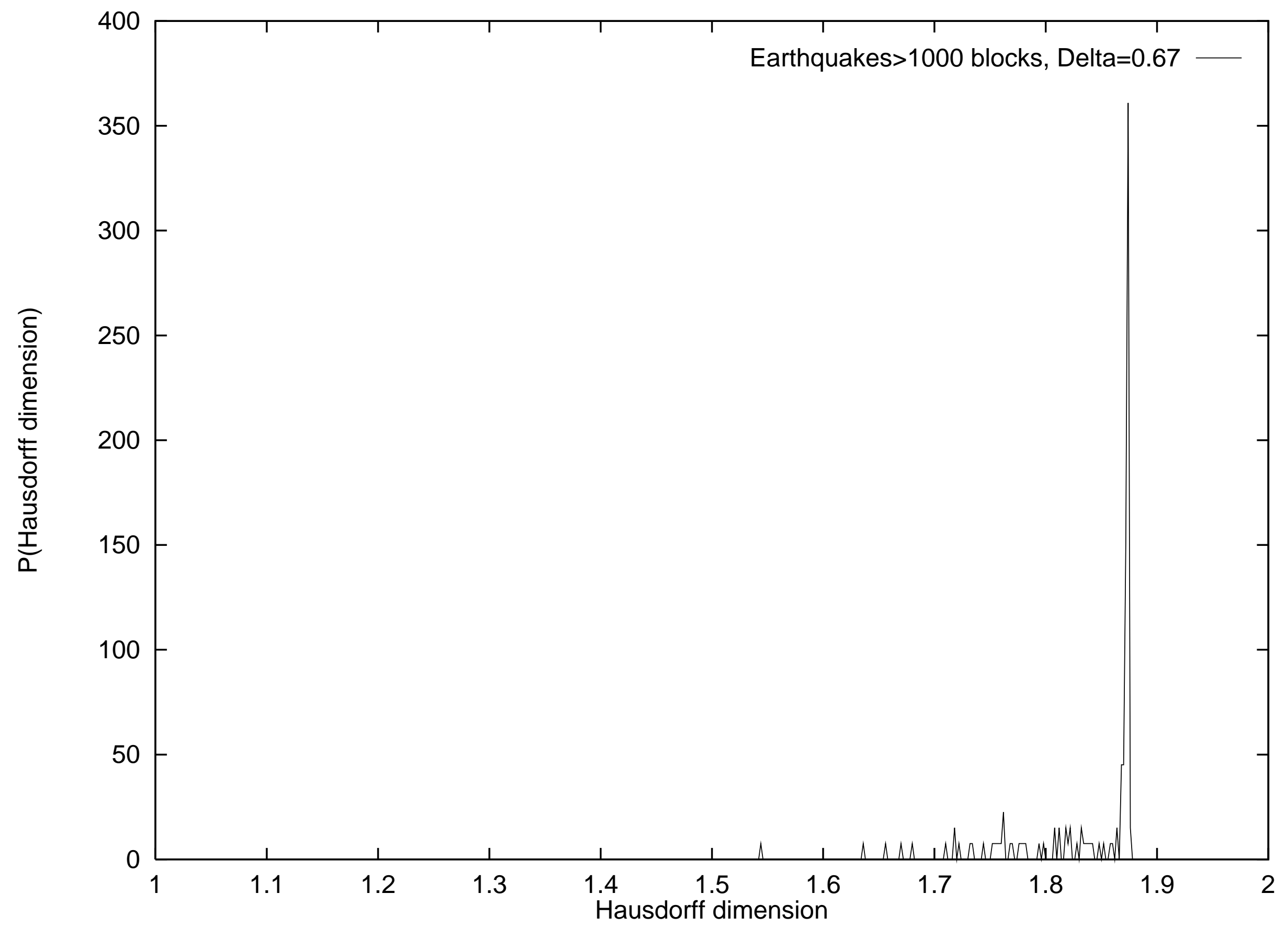




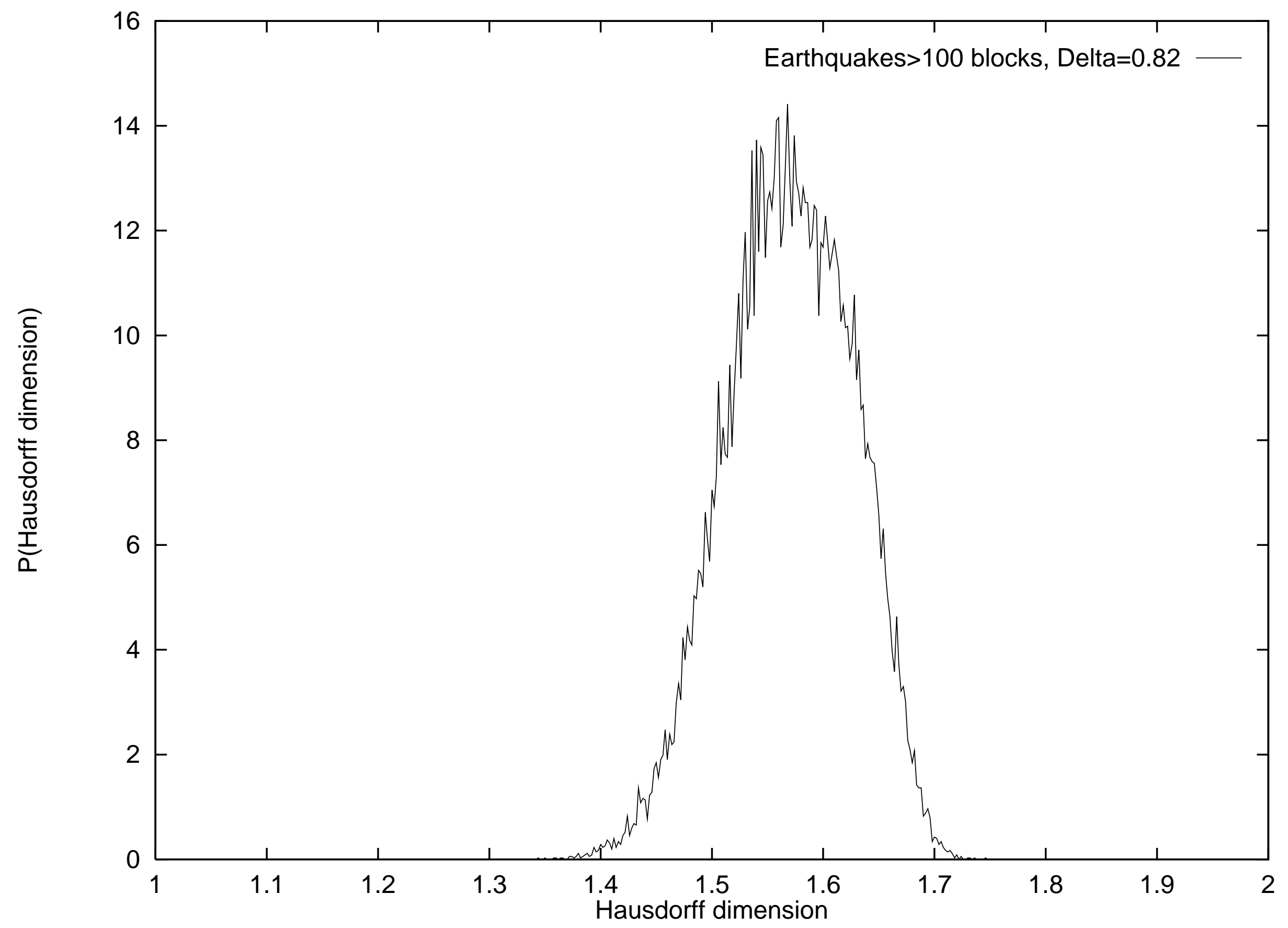




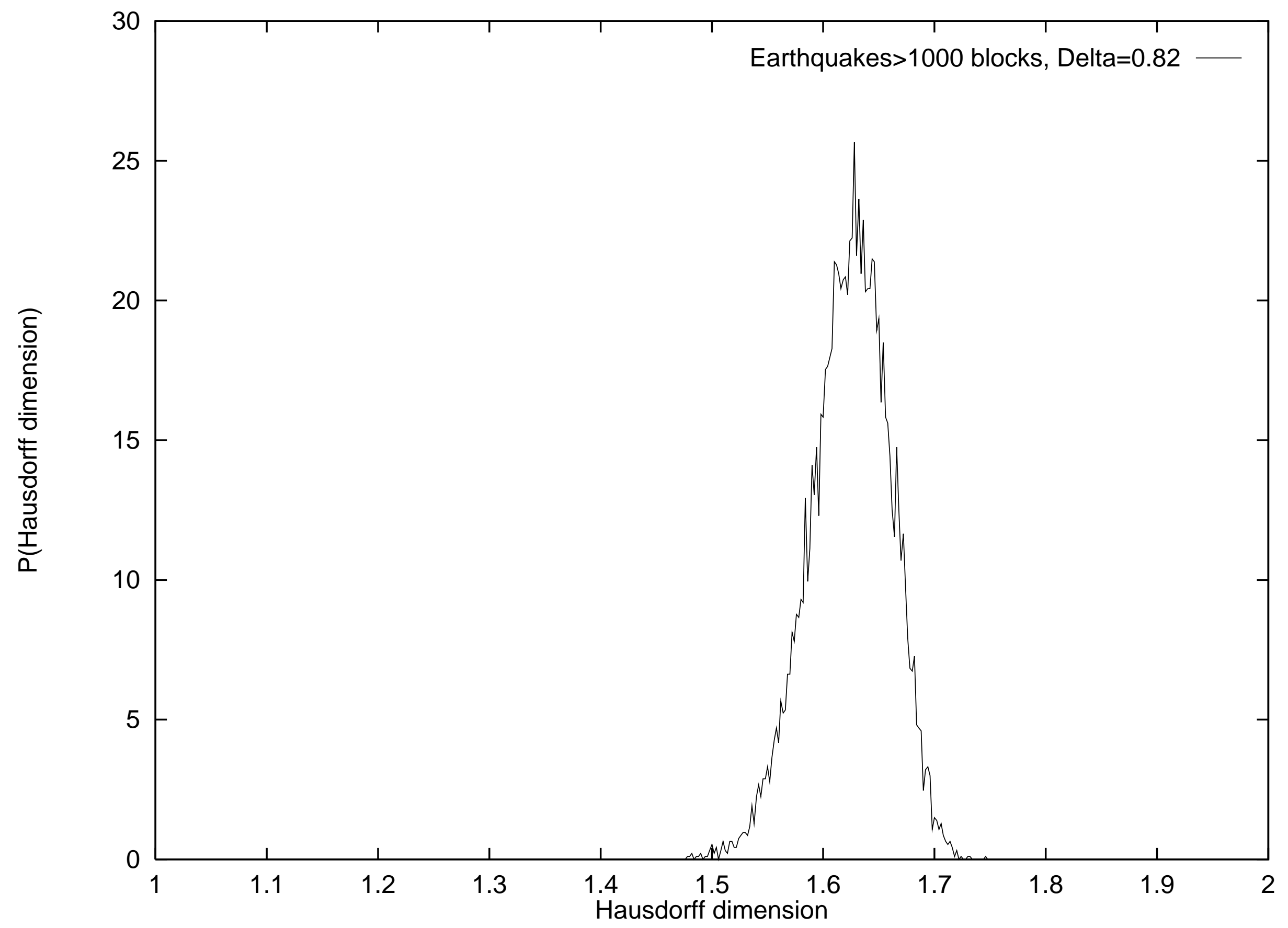




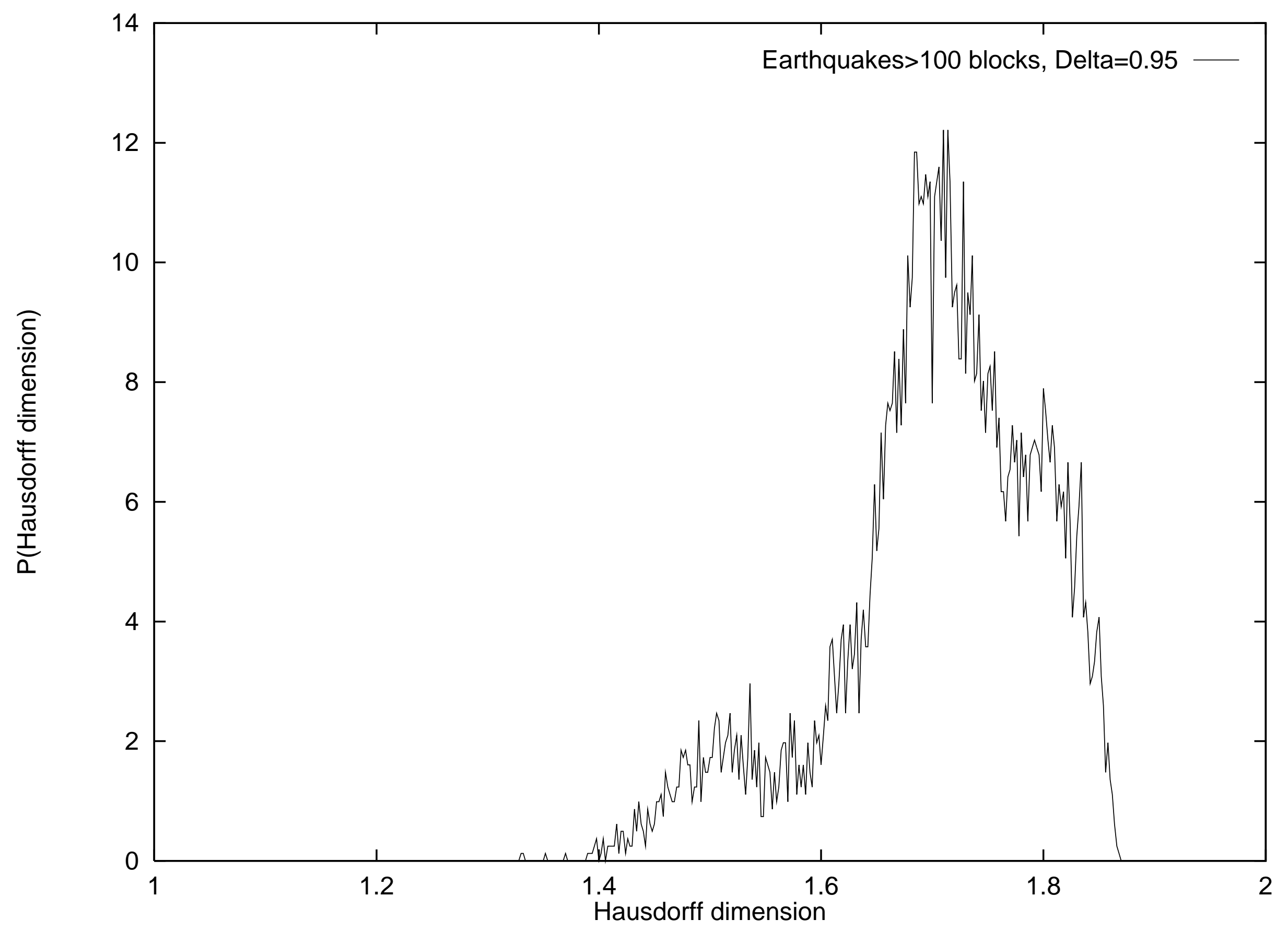




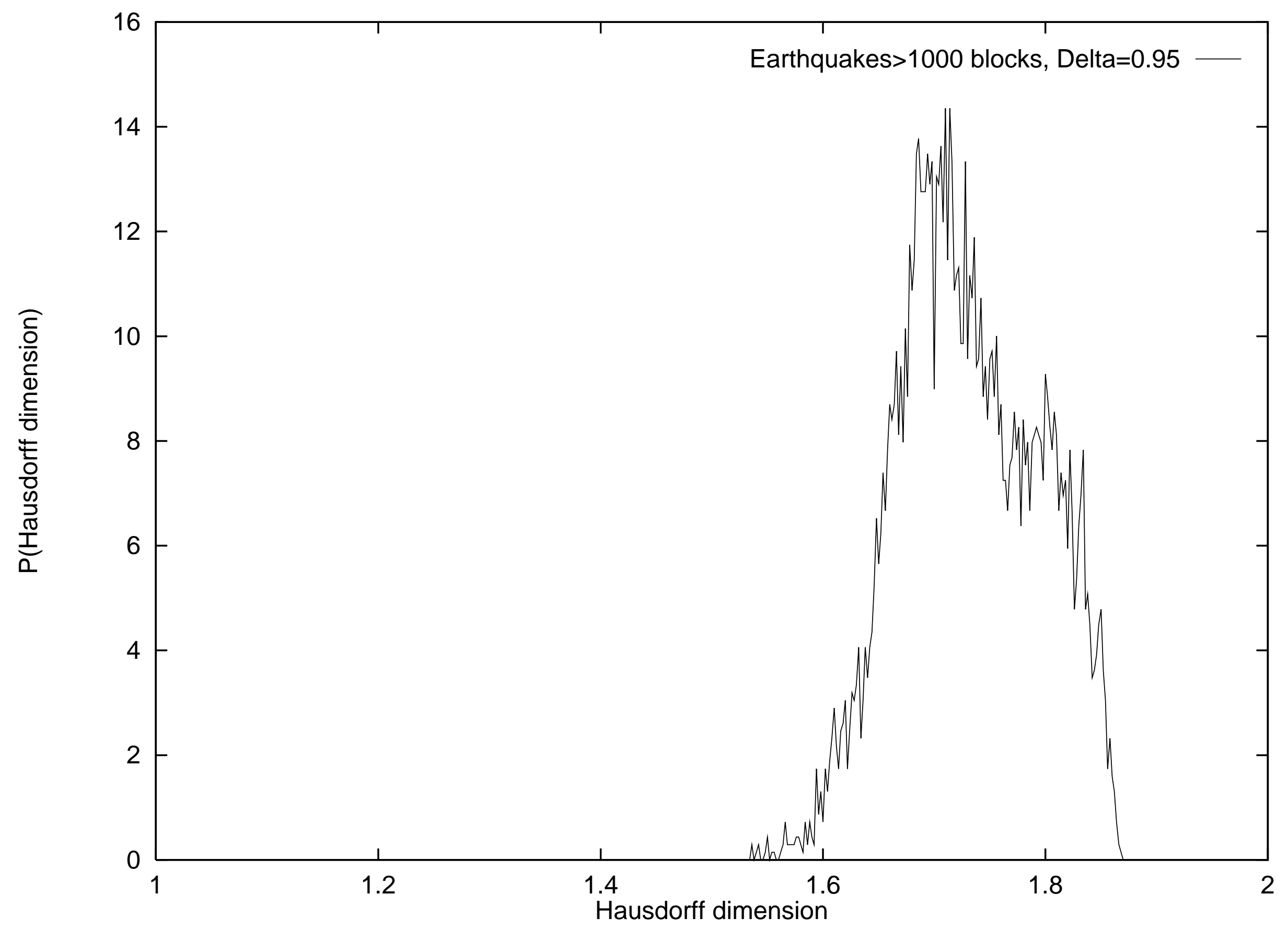




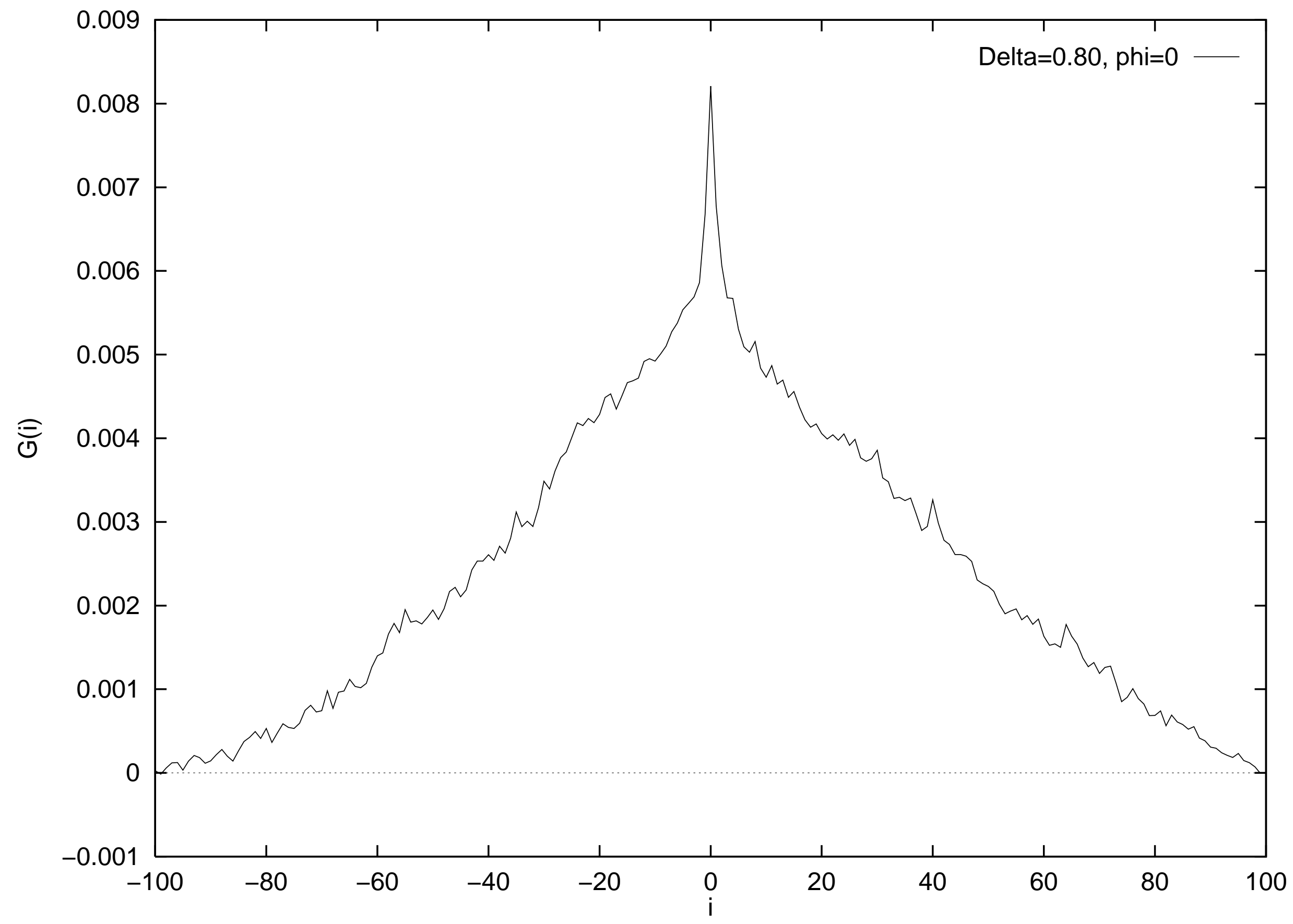




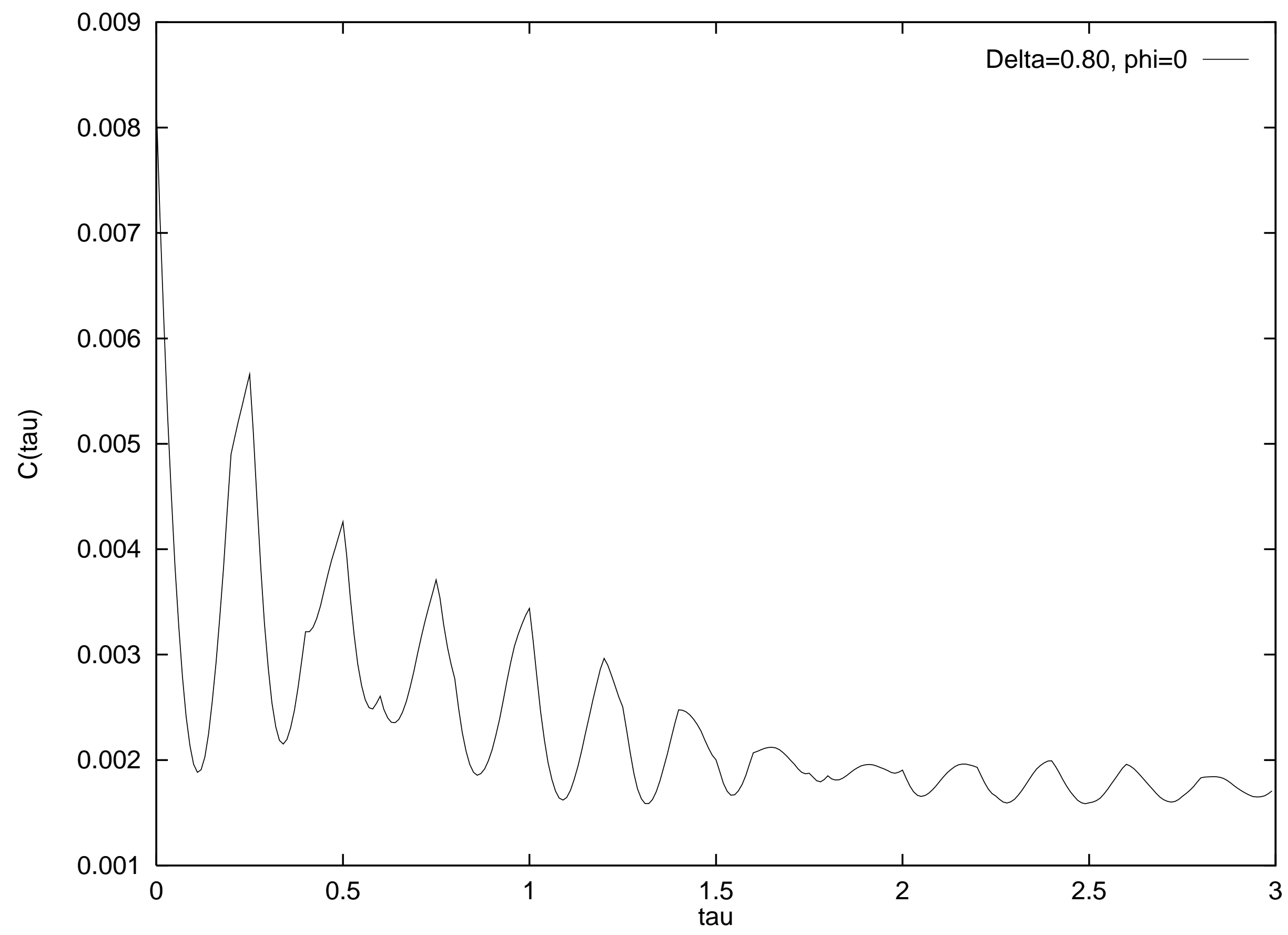

